\title{
DISTANCE TO SOLVABILITY/UNSOLVABILITY IN LINEAR OPTIMIZATION*
}

\author{
M. J. CÁNOVAS ${ }^{\dagger}$, M. A. LÓPEZ ${ }^{\ddagger}, J$. PARRA ${ }^{\dagger}$, AND F. J. TOLEDO ${ }^{\dagger}$
}

\begin{abstract}
In this paper we measure how much a linear optimization problem, in $\mathbb{R}^{n}$, has to be perturbed in order to lose either its solvability (i.e., the existence of optimal solutions) or its unsolvability property. In other words, if we consider as ill-posed those problems in the boundary of the set of solvable ones, then we can say that this paper deals with the associated distance to ill-posedness. Our parameter space is the set of all the linear semi-infinite programming problems with a fixed, but arbitrary, index set. In this framework, which includes as a particular case the ordinary linear programming, we obtain a formula for the distance from a solvable problem to unsolvability in terms of the nominal problem's coefficients. Moreover, this formula also provides the exact expression, or a lower bound, of the distance from an unsolvable problem to solvability. The relationship between the solvability and the primal-dual consistency is analyzed in the semi-infinite context, underlining the differences with the finite case.
\end{abstract}

Key words. stability, ill-posedness, distance to ill-posedness, solvability, duality, linear semiinfinite programming

AMS subject classifications. 65F22, 90C34, 15A39, 52A40

DOI. $10.1137 / 040612981$

1. Introduction. Different concepts of distance to ill-posedness have recently acquired remarkable prominence in different settings related to linear programming. Besides providing quantitative measures of the stability of a problem, they are related to several theoretical and numerical issues, namely, stability of the feasible set [3], [6], [19]; measures of conditioning [10], [17], [20]; complexity analysis of certain algorithms for computing solutions [9], [11]; size of the feasible set [3], [8]; metric regularity of mappings [6], [7], [15]; etc.

An instance of a problem is ill-posed with respect to a certain property if arbitrarily small perturbations of the data defining the problem instance can yield problem instances with and without the property. In this way, the respective boundaries of the sets of consistent problems (i.e., with nonempty solution set), bounded problems (i.e., with finite optimal value), or solvable problems (i.e., having optimal solutions) can be seen as examples of sets of ill-posed problems. The distance from a problem to any of these boundaries is referred to as its distance to ill-posedness with respect to the considered property.

The distance to ill-posedness with respect to consistency has been thoroughly studied, for example, in the contexts of conic linear systems [16], [18], [19] and linear semi-infinite inequality systems [3].

This paper is concerned with the distance to ill-posedness with respect to the boundedness and solvability of linear optimization problems, in $\mathbb{R}^{n}$, of the following

*Received by the editors August 6, 2004; accepted for publication (in revised form) May 12, 2005; published electronically January 6, 2006. This research has been partially supported by grants BFM2002-04114-C02 (01-02) from MCYT (Spain) and FEDER (E.U.), and GV04B-648 and GRUPOS04/79 from Generalitat Valenciana (Spain).

http://www.siam.org/journals/siopt/16-3/61298.html

†Operations Research Center, Miguel Hernández University of Elche, 03202 Elche (Alicante), Spain (canovas@umh.es, parra@umh.es, javier.toledo@umh.es).

${ }_{\ddagger}^{\ddagger}$ Department of Statistics and Operations Research, University of Alicante, 03071 Alicante, Spain (marco.antonio@ua.es). 
form:

$$
\begin{array}{lll}
\pi: & \text { Inf } & c^{\prime} x \\
& \text { s.t. } & a_{t}^{\prime} x \geq b_{t}, t \in T,
\end{array}
$$

where $c, x, a_{t} \in \mathbb{R}^{n}, b_{t} \in \mathbb{R}$, and $y^{\prime}$ denotes the transpose of $y \in \mathbb{R}^{n}$. The index set, $T$, of the constraint system, $\sigma=\left\{a_{t}^{\prime} x \geq b_{t}, t \in T\right\}$, is arbitrary. The feasible set of $\pi$ is denoted by $F$, its optimal value by $v$, and its optimal set by $F^{o p}$, adopting the convention $v=+\infty$ when $F=\emptyset$.

When $T$ is finite $\pi$ is nothing else but an ordinary linear programming problem, whereas $\pi$ is a linear semi-infinite programming problem when $T$ is infinite. In the latter case the problem of determining the distance to the ill-posedness with respect to the solvability is not reducible to the problem of determining the distance to the ill-posedness with respect the consistency of the combined system of primal and dual constraints [5], since the dual problem is infinite-dimensional and there might exist a duality gap (see [12, Chap. 8]). Nevertheless, in section 6 the relationship between solvability and primal-dual consistency is explored.

The parameter space of all the linear optimization problems $\pi=(c, \sigma)$ in the form (1), and whose constraint systems have the same index set $T$, is denoted by $\Pi$. When different problems are considered in $\Pi$, they and their associated elements will be distinguished by means of sub- or superscripts. Thus, if $\pi_{1}$ also belongs to $\Pi$, we write $\pi_{1}=\left(c^{1}, \sigma_{1}\right)$ and $\sigma_{1}:=\left\{\left(a_{t}^{1}\right)^{\prime} x \geq b_{t}^{1}, t \in T\right\}$, and its feasible set, optimal value, and optimal set are accordingly denoted by $F_{1}, v_{1}$, and $F_{1}^{o p}$, respectively.

$\Pi_{c}$ will denote the subset of $\Pi$ formed by all the consistent problems, while $\Pi_{i}:=$ $\Pi \backslash \Pi_{c}$ represents the subset of all the inconsistent problems. $\Pi_{b}$ denotes the subset of the bounded problems, and $\Pi_{s}$ the subset of the solvable ones, that is, those problems with nonempty optimal set $\left(F^{o p} \neq \emptyset\right)$. Obviously $\Pi_{s} \subset \Pi_{b} \subset \Pi_{c}$.

Associated with two arbitrary norms in $\mathbb{R}^{n}$ and $\mathbb{R}^{n+1}$, both denoted by $\|\cdot\|$, the extended distance $\delta: \Pi \times \Pi \rightarrow[0,+\infty]$ given by

$$
\delta\left(\pi_{1}, \pi\right):=\max \left\{\left\|c^{1}-c\right\|, d\left(\sigma_{1}, \sigma\right)\right\},
$$

where

$$
d\left(\sigma_{1}, \sigma\right):=\sup _{t \in T}\left\|\left(\begin{array}{c}
a_{t}^{1} \\
b_{t}^{1}
\end{array}\right)-\left(\begin{array}{c}
a_{t} \\
b_{t}
\end{array}\right)\right\| .
$$

This extended distance endows $\Pi$ with the topology of the uniform convergence of the coefficients vectors (see [12, Chapter 10] for details). The space $\Pi$ locally behaves as a normed space.

Given $\pi \in \Pi$ and $\widetilde{\Pi} \subset \Pi$, we will write, as usual,

$$
\delta(\pi, \widetilde{\Pi}):=\inf \{\delta(\pi, \widetilde{\pi}), \widetilde{\pi} \in \widetilde{\Pi}\} \in[0,+\infty] .
$$

If $\varnothing \neq \widetilde{\Pi}$ and $\pi \notin \widetilde{\Pi}$, one has $\delta(\pi, \widetilde{\Pi})=\delta(\pi, b d(\widetilde{\Pi}))$.

If $X$ is a subset of any topological space, $\operatorname{int}(X), \operatorname{cl}(X)$, and $b d(X)$ denote the interior set, the closure, and the boundary of $X$, respectively. By ext $(X)$ we represent the exterior of $X$, i.e., the complementary set of $\operatorname{cl}(X)$.

In $[4$, Thm. 1], it is proved that the set of ill-posed problems with respect to solvability, $b d\left(\Pi_{s}\right)$, coincides with $b d\left(\Pi_{b}\right)$. Moreover, this set is characterized there by means of some results which we gather, among other preliminaries, in section 2 . 
In fact, in order to achieve our goal of finding an expression for the distance to illposedness $\delta\left(\pi, b d\left(\Pi_{s}\right)\right)$, we appeal to a collection of results about the stability and well-posedness established in [2], [3], [4], [12], and [13]. In section 3 we provide (via Theorems 1 and 2) an explicit formula (5) for the distance to unsolvability from a solvable problem. Specifically, this formula consists of the minimum of two distances in $\mathbb{R}^{n}$ and $\mathbb{R}^{n+1}$, respectively, which depend only on the problem's data. We point out that the first of these distances turns out to be the distance to (primal) inconsistency for the given problem, whereas the second one is, as we prove in Theorem 6 , the distance to dual inconsistency. On the other hand, Theorems 3 and 4 establish that the previous formula can be extended to certain unsolvable problems. Theorem 1 gathers all the cases for which the formula holds. In the remaining cases we show that the right-hand side of (5) still stands as a lower bound for the distance to ill-posedness, and a general upper bound is also given (Theorem 5), again in terms of the problem's data. Section 4 provides more precise upper bounds under certain additional hypotheses. Section 5 is devoted to presenting some examples and counterexamples which delimit and illustrate the main results of the paper. Specifically, Examples 5 and 6 show the difficulties in providing a formula of $\delta\left(\pi, b d\left(\Pi_{s}\right)\right)$ when Theorem 1 does not apply. Section 6 approaches the ill-posedness with respect to the dual consistency and analyzes the relationship between the ill-posedness with respect to the solvability and with respect to primal-dual consistency. This section, together with section 7, integrates the contributions of the paper within the related literature on conditioning in linear optimization, paying attention to the backgrounds in the finite case (with $T$ finite) traced from [5], [10], and [20]. We emphasize the differences between the finite case and the general one ( $T$ arbitrary). These two last sections show how formula (5) generalizes to our semi-infinite context the corresponding result for finite solvable linear programming problems. Moreover, (5) extends to a certain subset of unsolvable problems, providing new results even for finite linear programming.

2. Preliminaries. This section presents the necessary notation and some basic definitions, results, and tools used in this paper. Given $\varnothing \neq X \subset \mathbb{R}^{k}, \operatorname{conv}(X)$ and cone $(X)$ denote the convex hull of $X$ and the conical convex hull of $X$, respectively. It is assumed that cone $(X)$ always contains the zero-vector $0_{k}$, and thus cone $(\varnothing)=\left\{0_{k}\right\}$. If $\Lambda \subset \mathbb{R}$, we introduce the set $\Lambda X:=\{\lambda x: \lambda \in \Lambda$ and $x \in X\}$.

If we consider any norm in $\mathbb{R}^{k},\|\cdot\|$, the corresponding open unit ball will be represented by $B$. Given a sequence $\left\{\mu_{r}\right\}, \lim _{r} \mu_{r}$ should be interpreted as $\lim _{r \rightarrow+\infty} \mu_{r}$.

Associated with $\pi=(c, \sigma)$, the following sets are relevant in our analysis:

$$
\begin{array}{ll}
A:=\operatorname{conv}\left(\left\{a_{t}, t \in T\right\}\right), & M:=\operatorname{cone}\left(\left\{a_{t}, t \in T\right\}\right)=\mathbb{R}_{+} A, \\
Z^{+}:=\operatorname{conv}\left(\left\{a_{t}, t \in T ; c\right\}\right), & Z^{-}:=\operatorname{conv}\left(\left\{a_{t}, t \in T ;-c\right\}\right), \\
C:=\operatorname{conv}\left(\left\{\left(\begin{array}{c}
a_{t} \\
b_{t}
\end{array}\right), t \in T\right\}\right), & H:=C+\mathbb{R}_{+}\left\{\left(\begin{array}{c}
0_{n} \\
-1
\end{array}\right)\right\},
\end{array}
$$

where $\mathbb{R}_{+}:=[0,+\infty[$. The sets $M$ and $H$ are, respectively, called the first moment cone and the hypographical set.

The existence of infinitely many coefficient vectors when $T$ is infinite gives rise to the following pathological subset of problems (see [3, sect. 3]):

$$
\Pi_{\infty}:=\left\{\pi \in \Pi \mid \delta\left(\pi, b d\left(\Pi_{c}\right)\right)=+\infty\right\} .
$$


The problems in $\Pi_{\infty}$ are characterized by the property that $\left(\begin{array}{c}0_{n} \\ 1\end{array}\right)$ belongs to the recession cone of $\operatorname{cl}(C)$; in other words, $\left(\begin{array}{c}0_{n} \\ 1\end{array}\right)=\lim _{r} \mu_{r} z^{r}$, with $\left\{z^{r}\right\}_{r=1}^{\infty} \subset C$ and $\left\{\mu_{r}\right\} \downarrow 0$. Moreover, $\Pi_{\infty} \subset \Pi_{i}$.

The following proposition gathers different results which are applied throughout the paper.

Proposition 1. Given $\pi=(c, \sigma) \in \Pi$, the following statements hold:

(i) $\left[2\right.$, Lem. 4.1] If $\pi \in \operatorname{int}\left(\Pi_{c}\right)$, then $\pi \in \operatorname{int}\left(\Pi_{s}\right)$ if and only if $c \in \operatorname{int}(M)$.

(ii) [12, Thm. 8.1(iv)] If $\pi \in \Pi_{b}$, then $c \in \operatorname{cl}(M)$.

(iii) $\left[4\right.$, Lem. 1(iv)] If $\pi \in \Pi_{c}$ and $c \in c l(M)$, then $\pi \in c l\left(\Pi_{s}\right)$.

(iv) $\left[4\right.$, Lem. 1(i) and Prop. 5] If $\pi \in b d\left(\Pi_{c}\right) \cup\left(\Pi_{i} \backslash \Pi_{\infty}\right)$, then $0_{n} \in \operatorname{cl}(A)$.

(v) $\left[4\right.$, Lem. 1(ii)] If $\pi \in b d\left(\Pi_{c}\right) \cap \Pi_{i}$, then $0_{n} \in b d(A)$.

(vi) [12, Thm. 6.3] If $\pi \in \Pi_{i}$ and $M=\mathbb{R}^{n}$, then $\pi \in$ int $\left(\Pi_{i}\right)$.

Along the lines of [8] and [10] (which deal with conic linear systems), $b d\left(\Pi_{c}\right)$ is considered as the set of ill-posed problems with respect to the consistency, and according to [19], the distance to ill-posedness is $\delta\left(\pi, b d\left(\Pi_{c}\right)\right)$. The following proposition describes the position of $\pi \in \Pi$ relative to $b d\left(\Pi_{c}\right)$ in terms of the relative position between $0_{n+1}$ and the boundary of the hypographical set, $b d(H)$.

Proposition 2 (see [3, Thms. 4, 5, and 6]). Let $\pi \in \Pi \backslash \Pi_{\infty}$. Then, the following statements hold:

(i) $\pi \in \operatorname{int}\left(\Pi_{i}\right) \Leftrightarrow 0_{n+1} \in \operatorname{int}(H)$;

(ii) $\pi \in \operatorname{int}\left(\Pi_{c}\right) \Leftrightarrow 0_{n+1} \in \operatorname{ext}(H)$;

(iii) $\pi \in b d\left(\Pi_{c}\right) \Leftrightarrow 0_{n+1} \in b d(H)$;

(iv) $\delta\left(\pi, b d\left(\Pi_{c}\right)\right)=d\left(0_{n+1}, b d(H)\right)$.

Observe that (iv) translates the problem of measuring the distance to ill-posedness with respect to the consistency, posed in the infinite-dimensional space $\Pi$, into the problem of calculating a distance in the $(n+1)$-dimensional Euclidean space.

The following proposition describes the position of $\pi \in \operatorname{int}\left(\Pi_{c}\right)$ relative to $b d\left(\Pi_{s}\right)$ in terms of the relative position between $0_{n}$ and the boundary of the set $Z^{-}$.

Proposition 3 (see [4, Thm. 2]). Given $\pi \in \operatorname{int}\left(\Pi_{c}\right)$, one has

(i) $\pi \in \operatorname{int}\left(\Pi_{s}\right) \Leftrightarrow 0_{n} \in \operatorname{int}\left(Z^{-}\right)$;

(ii) $\pi \in b d\left(\Pi_{s}\right) \Leftrightarrow 0_{n} \in b d\left(Z^{-}\right)$;

(iii) $\pi \in \operatorname{ext}\left(\Pi_{s}\right) \Leftrightarrow 0_{n} \in \operatorname{ext}\left(Z^{-}\right)$.

The next result characterizes those problems that, being ill-posed with respect to the consistency, are also ill-posed with respect to the solvability.

Proposition 4 (see [4, Thm. 3]). Let $\pi \in b d\left(\Pi_{c}\right)$. Then $\pi \in b d\left(\Pi_{s}\right)$ if and only if either $\pi \in \operatorname{cl}\left(b d\left(\Pi_{c}\right) \cap \Pi_{c}\right)$ or $0_{n} \in b d\left(Z^{+}\right)$.

The following proposition explores the relationship between the condition $\pi \in$ $c l\left(b d\left(\Pi_{c}\right) \cap \Pi_{c}\right)$ and the data-set $C$.

Proposition 5 (see [4, Thm. 4]). Let $\pi \in b d\left(\Pi_{c}\right)$. If $\pi \in \operatorname{cl}\left(b d\left(\Pi_{c}\right) \cap \Pi_{c}\right)$, then $0_{n+1} \in b d(C)$. The converse statement holds when $\left\{b_{t}, t \in T\right\}$ is bounded.

The following proposition, which is a straightforward consequence of Propositions 3,4 , and 5 , provides a complete characterization of the ill-posed problems whose constraint systems have a bounded right-hand side.

Proposition 6 (see [4, Thm. 5]). Let $\pi \in \Pi$, and suppose that the set $\left\{b_{t}, t \in T\right\}$ is bounded. Then, $\pi \in b d\left(\Pi_{s}\right)$ if and only if some of the following statements hold:

(i) $0_{n+1} \in \operatorname{ext}(H)$ and $0_{n} \in b d\left(Z^{-}\right)$;

(ii) $0_{n+1} \in b d(H) \cap b d(C)$;

(iii) $0_{n+1} \in b d(H)$ and $0_{n} \in b d\left(Z^{+}\right)$.

The following results admit straightforward proofs. 
Proposition 7. The sets $M, A, Z^{+}$, and $Z^{-}$satisfy the following relations:

$$
c \in \operatorname{int}(M) \Leftrightarrow 0_{n} \in \operatorname{int}\left(Z^{-}\right) \text {and }-c \in \operatorname{int}(M) \Leftrightarrow 0_{n} \in \operatorname{int}\left(Z^{+}\right) .
$$

In particular, if $c=0_{n}$, then $Z^{+}=Z^{-}$and

$$
0_{n} \in \operatorname{int}\left(Z^{-}\right) \Leftrightarrow 0_{n} \in \operatorname{int}(A) \Leftrightarrow 0_{n} \in \operatorname{int}(M) \Leftrightarrow M=\mathbb{R}^{n} .
$$

Proposition 8. Let $S \neq \varnothing$ be an arbitrary index set and let $X:=\left\{x^{s}, s \in S\right\}$ and $Y:=\left\{y^{s}, s \in S\right\}$ be two subsets of $\mathbb{R}^{k}$ such that $\sup _{s \in S}\left\|x^{s}-y^{s}\right\| \leq \varepsilon$ for certain $\varepsilon \geq 0$. Then one has the following:

(i) If $\rho \mathrm{cl}(B) \subset \operatorname{cl}(\operatorname{conv}(X))$ for some $\rho \geq \varepsilon$, then

$$
(\rho-\varepsilon) \operatorname{cl}(B) \subset \operatorname{cl}(\operatorname{conv}(Y)) .
$$

(ii) If $\rho c l(B) \cap c l(\operatorname{conv}(X))=\varnothing$ for some $\rho \geq \varepsilon$, then

$$
(\rho-\varepsilon) \operatorname{cl}(B) \cap \operatorname{cl}(\operatorname{conv}(Y))=\varnothing .
$$

Figure 1 summarizes the information we have already presented about the structure of $\Pi \backslash \Pi_{\infty}$ in relation to the properties of consistency and solvability.

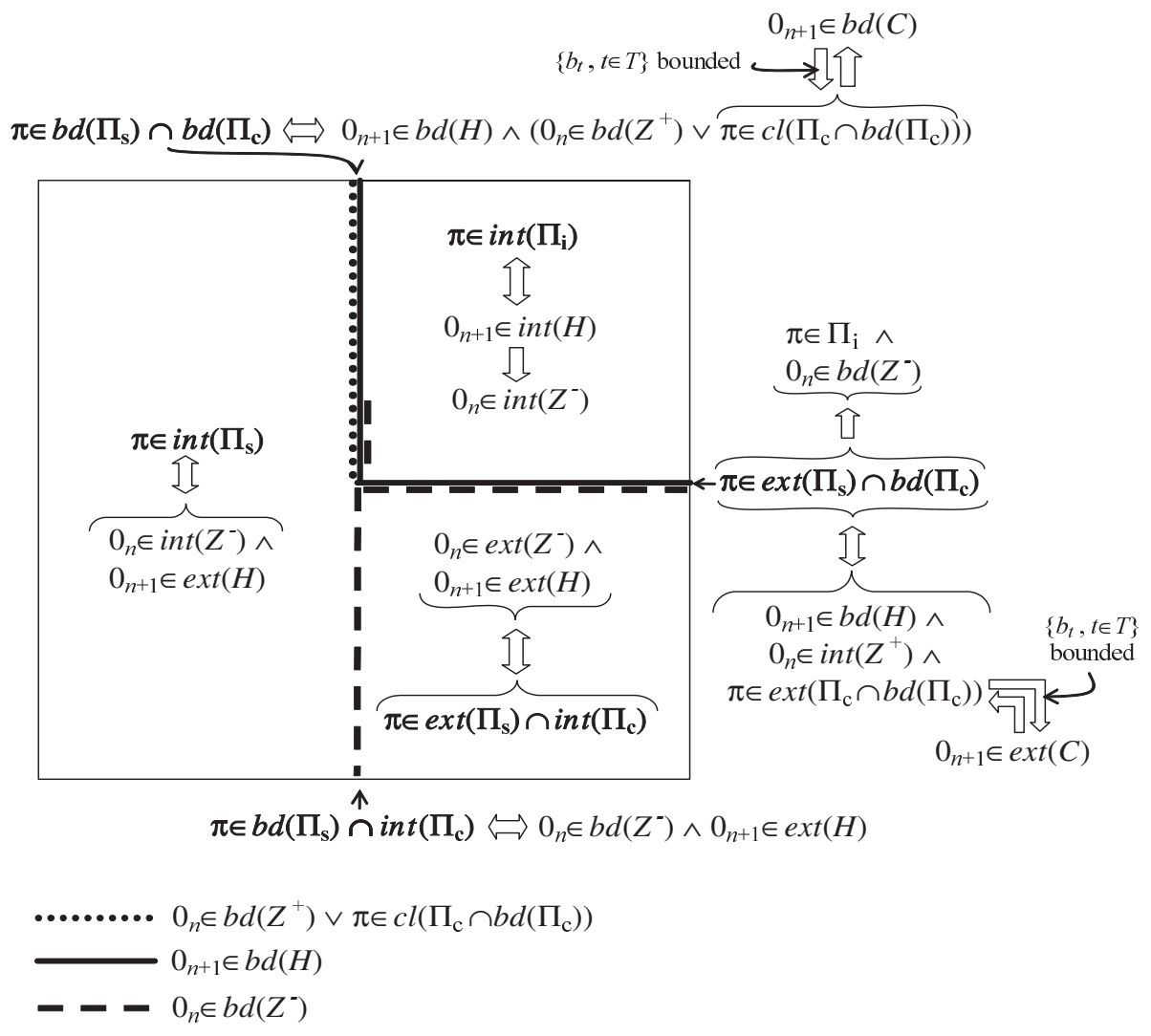

FiG. 1. Structure of $\Pi \backslash \Pi_{\infty}$. 
Throughout the paper we assume that the norm $\|\cdot\|$ considered in $\mathbb{R}^{n+1}$ verifies

$$
\left\|\left(\begin{array}{l}
a \\
b
\end{array}\right)\right\|=\left\|\left(\begin{array}{c}
a \\
-b
\end{array}\right)\right\| \text { for all }\left(\begin{array}{l}
a \\
b
\end{array}\right) \in \mathbb{R}^{n+1} .
$$

Observe that any $p$-norm, but not any norm (see [21, Thm. 15.2]), verifies this condition. In $\mathbb{R}^{n}$ the norm (also denoted by $\|\cdot\|$ ) given by

$$
\|a\|:=\left\|\left(\begin{array}{l}
a \\
0
\end{array}\right)\right\| \text { for all } a \in \mathbb{R}^{n}
$$

will be considered. Note that if the norm considered in $\mathbb{R}^{n+1}$ is a $p$-norm, $p \in[1,+\infty]$, the norm in $\mathbb{R}^{n}$ is also a $p$-norm (with the same $p$ ).

Remark 1. Property (3) implies that $\left\|\left(\begin{array}{c}a \\ b_{1}\end{array}\right)\right\| \leq\left\|\left(\begin{array}{c}a \\ b_{2}\end{array}\right)\right\|$ when $\left|b_{1}\right| \leq\left|b_{2}\right|$. The proof is a straightforward consequence of the fact that $\left(\begin{array}{c}a \\ b_{1}\end{array}\right)$ is a convex combination of $\left(\begin{array}{c}a \\ b_{2}\end{array}\right)$ and $\left(\begin{array}{c}a \\ -b_{2}\end{array}\right)$.

3. Distance to solvability/unsolvability. In the present section we approach the problem of determining the distance to ill-posedness, $\delta\left(\pi, b d\left(\Pi_{s}\right)\right)$, for a given problem $\pi \in \Pi \backslash \Pi_{\infty}$. The case $\pi \in \Pi_{\infty}$ is obvious as far as $\Pi_{\infty} \subset \Pi_{i}$, and then $\delta\left(\pi, b d\left(\Pi_{s}\right)\right) \geq \delta\left(\pi, b d\left(\Pi_{c}\right)\right)=+\infty$. We will analyze different cases obtaining either an exact expression (see Theorem 1) or lower and upper bounds (see Theorem 5 and the subsequent results) for this distance in terms of the problem's data.

The following theorem is the main result in this paper and partially synthesizes the statements of Theorems 2, 3, 4, and 5 in relation to the exact formula for the distance to ill-posedness mentioned in the previous paragraph.

Theorem 1. Let $\pi=(c, \sigma) \in \Pi \backslash \Pi_{\infty}$. Suppose that at least one of the following conditions holds:

(i) $\pi \in \operatorname{cl}\left(\Pi_{s}\right)$;

(ii) $\pi \in \operatorname{ext}\left(\Pi_{s}\right)$ and $d\left(0_{n+1}, b d(H)\right) \neq d\left(0_{n}, b d\left(Z^{-}\right)\right)$;

(iii) $d\left(0_{n+1}, b d(H)\right)=d\left(0_{n}, b d\left(Z^{-}\right)\right) \geq\|c\|$.

Then one has

$$
\delta\left(\pi, b d\left(\Pi_{s}\right)\right)=\min \left\{d\left(0_{n+1}, b d(H)\right), d\left(0_{n}, b d\left(Z^{-}\right)\right)\right\} .
$$

Proof. (i) See Theorem 2.

(ii) $\pi \in \operatorname{ext}\left(\Pi_{s}\right)$ implies either $\pi \in \operatorname{int}\left(\Pi_{c}\right)$, in which case Theorem 3 applies, or $\pi \in \Pi_{i}$, and Theorem 4 applies, since otherwise one has $\pi \in b d\left(\Pi_{c}\right) \cap \Pi_{c}$ and Proposition 4 yields a contradiction.

(iii) See Theorem 5.

Remark 2. Note that, by virtue of Proposition 2, one has $d\left(0_{n+1}, b d(H)\right)=$ $\delta\left(\pi, b d\left(\Pi_{c}\right)\right)$; that is, the distance to ill-posedness with respect to the solvability depends on the distance to ill-posedness with respect to the consistency, as one would expect.

Remark 3. Formula (5) for the distance to ill-posedness does not hold, in general, in the remaining case corresponding to the problems $\pi \in \operatorname{ext}\left(\Pi_{s}\right)$ such that

$$
d\left(0_{n+1}, b d(H)\right)=d\left(0_{n}, b d\left(Z^{-}\right)\right)<\|c\|,
$$

even in ordinary linear programming in $\mathbb{R}$ with "few" constraints, as we can see in Examples 3, 4, and 5. Nevertheless, in this case one has, as a straightforward consequence of Theorem 5 , that

$$
d\left(0_{n+1}, b d(H)\right) \leq \delta\left(\pi, b d\left(\Pi_{s}\right)\right) \leq\|c\| .
$$


The next lemma will be used later on.

LEMMA 1. Given $\pi \in \Pi$, one has the following:

(i) If $0_{n} \in b d(A)$ and $c \in c l(M)$, then $0_{n} \in b d\left(Z^{+}\right)$.

(ii) If $\pi \in b d\left(\Pi_{c}\right)$ and $c \in c l(M)$, then $\pi \in b d\left(\Pi_{s}\right)$.

(iii) If $0_{n} \in \operatorname{int}\left(Z^{-} \cap Z^{+}\right)$, then $0_{n} \in \operatorname{int}(A)$.

Proof. (i) Since $0_{n} \in b d(A)$, we have $0_{n} \in \operatorname{cl}\left(Z^{+}\right)$. If $0_{n} \in \operatorname{int}\left(Z^{+}\right)$, then $-c \in \operatorname{int}(M)$ (see Proposition 7) and, since $c \in c l(M)$ by assumption, Theorem 6.1 in [21] ensures that $0_{n} \in \operatorname{int}(M)$ and, then, we get the contradiction $0_{n} \in \operatorname{int}(A)$ (again by Proposition 7).

(ii) We distinguish two cases. If $\pi \in \Pi_{c}$, one has $\pi \in b d\left(\Pi_{s}\right)$ by virtue of Proposition 4. If $\pi \in \Pi_{i}$, then $0_{n} \in b d(A)$ from Proposition 1(v); thus, the previous statement implies $0_{n} \in b d\left(Z^{+}\right)$and therefore $\pi \in b d\left(\Pi_{s}\right)$, again by virtue of Proposition 4 .

(iii) Under this hypothesis we have, by Proposition 7, $c \in \operatorname{int}(M)$ and $-c \in$ $\operatorname{int}(M)$; thus, by convexity of $\operatorname{int}(M), 0_{n} \in \operatorname{int}(M)$ and Proposition 7 again leads us to $0_{n} \in \operatorname{int}(A)$.

The following theorem establishes that (5) is valid for a problem in the closure of the set of solvable problems.

THEOREM 2. Let $\pi \in \operatorname{cl}\left(\Pi_{s}\right)$. Then (5) holds, i.e.,

$$
\delta\left(\pi, b d\left(\Pi_{s}\right)\right)=\min \left\{d\left(0_{n+1}, b d(H)\right), d\left(0_{n}, b d\left(Z^{-}\right)\right)\right\}
$$

Proof. First let us consider the case $\pi \in b d\left(\Pi_{s}\right)$ and let us see that the righthand side in (5) is zero. Indeed, if $\pi \in \operatorname{int}\left(\Pi_{c}\right)$, Proposition 3(ii) ensures that $d\left(0_{n}, b d\left(Z^{-}\right)\right)=0$, and if $\pi \in b d\left(\Pi_{c}\right)$, Proposition 2(iii) guarantees that $d\left(0_{n+1}, b d(H)\right)$ $=0$.

From now on we will suppose $\pi \in \operatorname{int}\left(\Pi_{s}\right)$. In order to establish the $\leq$ inequality, let us see that the following inequalities are simultaneously satisfied:

(a) $\delta\left(\pi, b d\left(\Pi_{s}\right)\right) \leq d\left(0_{n+1}, b d(H)\right)$, and

(b) $\delta\left(\pi, b d\left(\Pi_{s}\right)\right) \leq d\left(0_{n}, b d\left(Z^{-}\right)\right)$.

Since $\pi \in \operatorname{int}\left(\Pi_{s}\right)$, we have

$$
\delta\left(\pi, b d\left(\Pi_{s}\right)\right) \leq \delta\left(\pi, b d\left(\Pi_{c}\right)\right)=d\left(0_{n+1}, b d(H)\right)
$$

and (a) holds. On the other hand, (b) is trivial if $Z^{-}=\mathbb{R}^{n}$. Otherwise, the distance $d\left(0_{n}, b d\left(Z^{-}\right)\right)$will be attained at certain $a \in b d\left(Z^{-}\right)$. Consequently we have $0_{n} \in$ $b d\left(Z^{-}-a\right)$.

If we consider the problem $\pi_{0}:=\left(c+a, \sigma_{0}\right)$, where

$$
\sigma_{0}:=\left\{\left(a_{t}-a\right)^{\prime} x \geq b_{t}, t \in T\right\},
$$

then $Z_{0}^{-}=Z^{-}-a$ and Proposition 7 entails $c+a \notin \operatorname{int}\left(M_{0}\right)$ (with $M_{0}=\operatorname{cone}\left(\left\{a_{t}-a\right.\right.$, $t \in T\}))$. Now Proposition 1(i) ensures that $\pi_{0} \notin i n t\left(\Pi_{s}\right)$. Therefore

$$
\delta\left(\pi, b d\left(\Pi_{s}\right)\right) \leq \delta\left(\pi, \pi_{0}\right)=\|a\|
$$

which establishes (b).

Now let $\alpha:=\min \left\{d\left(0_{n+1}, b d(H)\right), d\left(0_{n}, b d\left(Z^{-}\right)\right)\right\}$, and take as before a point $a \in b d\left(Z^{-}\right)$at which the distance $d\left(0_{n}, b d\left(Z^{-}\right)\right)$is attained, supposing for the moment that $Z^{-} \neq \mathbb{R}^{n}$. From (a) and (b) we have $\delta\left(\pi, b d\left(\Pi_{s}\right)\right) \leq \alpha$ (for all $\left.\pi \in \operatorname{int}\left(\Pi_{s}\right)\right)$. To see that equality (5) holds, it is sufficient to prove that every problem $\pi_{1}:=\left(c^{1}, \sigma_{1}\right) \in \Pi$ such that $\delta\left(\pi, \pi_{1}\right)<\alpha$ is still in $\operatorname{int}\left(\Pi_{s}\right)$. 
Take $\pi_{1} \in \Pi$ in the previous conditions. Since $\pi \in \operatorname{int}\left(\Pi_{c}\right)$ and $\delta\left(\pi, \pi_{1}\right)<$ $d\left(0_{n+1}, b d(H)\right)$, we have $\pi_{1} \in \operatorname{int}\left(\Pi_{c}\right)$ (Proposition 2). On the other hand, since $\pi \in \operatorname{int}\left(\Pi_{s}\right)$, one has $c \in \operatorname{int}(M)$ by virtue of Proposition 1(i), and then $0_{n} \in$ $\operatorname{int}\left(Z^{-}\right)$(by Proposition 7). Indeed $\|a\| \operatorname{cl}(B) \subset \operatorname{cl}\left(Z^{-}\right)$and $\alpha \leq\|a\|$. Writing $\delta\left(\pi, \pi_{1}\right)=\alpha-\varepsilon$ for some $0<\varepsilon \leq \alpha$, Proposition 8(i) entails $\varepsilon c l(B) \subset \operatorname{cl}\left(Z_{1}^{-}\right)$, and then $0_{n} \in \operatorname{int}\left(\operatorname{cl}\left(Z_{1}^{-}\right)\right)=\operatorname{int}\left(Z_{1}^{-}\right)$. So, taking into account that $\pi_{1} \in \operatorname{int}\left(\Pi_{c}\right)$, Proposition 3(i) ensures that $\pi_{1} \in$ int $\left(\Pi_{s}\right)$.

Finally, in the case $Z^{-}=\mathbb{R}^{n}$ one has $\alpha=d\left(0_{n+1}, b d(H)\right)$, and then if $\delta\left(\pi, \pi_{1}\right)<$ $\alpha$, one has $\pi_{1} \in \operatorname{int}\left(\Pi_{c}\right)$ and, trivially, $0_{n} \in \operatorname{int}\left(Z_{1}^{-}\right)$, because $Z_{1}^{-}=\mathbb{R}^{n}$ (by Proposition 8). Thus $\pi_{1} \in \operatorname{int}\left(\Pi_{s}\right)$.

Examples 1 and 2 illustrate formula (5) for the problem $\pi \in \operatorname{cl}\left(\Pi_{s}\right)$. In the first example, one has $\delta\left(\pi, b d\left(\Pi_{s}\right)\right)=d\left(0_{n}, b d\left(Z^{-}\right)\right)<d\left(0_{n+1}, b d(H)\right)$, while in the second example $\delta\left(\pi, b d\left(\Pi_{s}\right)\right)=d\left(0_{n+1}, b d(H)\right)<d\left(0_{n}, b d\left(Z^{-}\right)\right)$. In both cases, a perturbation for obtaining a problem where the distance to ill-posedness is attained will be indicated.

Now we approach the distance to ill-posedness for the problem $\pi \in \operatorname{ext}\left(\Pi_{s}\right) \cap$ $\operatorname{int}\left(\Pi_{c}\right)$.

ThEOREM 3. Let $\pi \in \operatorname{ext}\left(\Pi_{s}\right) \cap \operatorname{int}\left(\Pi_{c}\right)$. Then

(i) $d\left(0_{n}, b d\left(Z^{-}\right)\right) \leq d\left(0_{n+1}, b d(H)\right)$;

(ii) $\delta\left(\pi, b d\left(\Pi_{s}\right)\right) \geq d\left(0_{n}, b d\left(Z^{-}\right)\right)$;

(iii) if $d\left(0_{n}, b d\left(Z^{-}\right)\right)<d\left(0_{n+1}, b d(H)\right)$, one has

$$
\delta\left(\pi, b d\left(\Pi_{s}\right)\right)=d\left(0_{n}, b d\left(Z^{-}\right)\right) .
$$

Proof. (i) Since $\pi \in \operatorname{ext}\left(\Pi_{s}\right) \cap \operatorname{int}\left(\Pi_{c}\right)$, Proposition 3(iii) ensures that $0_{n} \in$ $\operatorname{ext}\left(Z^{-}\right)$. Take $a \in b d\left(Z^{-}\right)$such that $d\left(0_{n}, b d\left(Z^{-}\right)\right)=\|a\|$.

For every $\left(\frac{\bar{a}}{b}\right) \in b d(H)$ we have $\bar{a} \in \operatorname{cl}(A) \subset \operatorname{cl}\left(Z^{-}\right)$and $\left\|\left(\frac{\bar{a}}{b}\right)\right\| \geq\|\bar{a}\| \geq\|a\|$ (see Remark 1). The arbitrariness of $\left(\frac{\bar{a}}{b}\right)$ entails $d\left(0_{n+1}, b d(H)\right) \geq\|a\|$ as we aimed to prove.

(ii) $\pi \in \operatorname{ext}\left(\Pi_{s}\right) \cap \operatorname{int}\left(\Pi_{c}\right)$ entails, by virtue of Propositions 2(ii) and 3(iii), $0_{n+1} \in \operatorname{ext}(H)$ and $0_{n} \in \operatorname{ext}\left(Z^{-}\right)$. Let $\pi_{1} \in \Pi$ with $\delta\left(\pi_{1}, \pi\right)<d\left(0_{n}, b d\left(Z^{-}\right)\right)$; then Proposition 8(ii) and part (i) ensure that $0_{n} \in \operatorname{ext}\left(Z_{1}^{-}\right)$and $0_{n+1} \in \operatorname{ext}\left(H_{1}\right)$. Now, again by Propositions 2(ii) and 3(iii), $\pi_{1} \in \operatorname{ext}\left(\Pi_{s}\right) \cap \operatorname{int}\left(\Pi_{c}\right)$ and therefore $\delta\left(\pi, b d\left(\Pi_{s}\right)\right) \geq d\left(0_{n}, b d\left(Z^{-}\right)\right)$.

(iii) From (ii) we need only prove $\delta\left(\pi, b d\left(\Pi_{s}\right)\right) \leq d\left(0_{n}, b d\left(Z^{-}\right)\right)$. Take again $a \in b d\left(Z^{-}\right)$such that $d\left(0_{n}, b d\left(Z^{-}\right)\right)=\|a\|$ and consider the same perturbation as in the proof of Theorem 2; i.e., we consider the problem $\pi_{0}=\left(c+a, \sigma_{0}\right)$, where

$$
\sigma_{0}:=\left\{\left(a_{t}-a\right)^{\prime} x \geq b_{t}, t \in T\right\} .
$$

On the one hand, we have $0_{n} \in b d\left(Z_{0}^{-}\right)=b d\left(Z^{-}-a\right)$. On the other hand, since $\pi \in \operatorname{int}\left(\Pi_{c}\right)$, Proposition 2(ii) guarantees that $0_{n+1} \in \operatorname{ext}(H)$, and the fact that $\delta\left(\pi, \pi_{0}\right)=\|a\|<d\left(0_{n+1}, b d(H)\right)$ by the current assumption, together with Proposition 8(ii), ensures that $0_{n+1} \in \operatorname{ext}\left(H_{0}\right)$. Again by virtue of Proposition 2(ii) one has $\pi_{0} \in \operatorname{int}\left(\Pi_{c}\right)$. Therefore $\pi_{0} \in b d\left(\Pi_{s}\right)$ by Proposition 3(ii) and, consequently, $\delta\left(\pi, b d\left(\Pi_{s}\right)\right) \leq \delta\left(\pi, \pi_{0}\right)=\|a\|$.

Example 3 shows that formula (5) for the problem $\pi \in \operatorname{ext}\left(\Pi_{s}\right) \cap \operatorname{int}\left(\Pi_{c}\right)$ does not hold in general when $d\left(0_{n}, b d\left(Z^{-}\right)\right)=d\left(0_{n+1}, b d(H)\right)$.

The next result is devoted to approaching the distance $\delta\left(\pi, b d\left(\Pi_{s}\right)\right)$ for the problem $\pi \in \Pi_{i} \backslash \Pi_{\infty}$. 
Theorem 4. Let $\pi \in \Pi_{i} \backslash \Pi_{\infty}$. Then

(i) $d\left(0_{n+1}, b d(H)\right) \leq d\left(0_{n}, b d\left(Z^{-}\right)\right)$;

(ii) $\delta\left(\pi, b d\left(\Pi_{s}\right)\right) \geq d\left(0_{n+1}, b d(H)\right)$;

(iii) if $d\left(0_{n+1}, b d(H)\right)<d\left(0_{n}, b d\left(Z^{-}\right)\right)$, one has

$$
\delta\left(\pi, b d\left(\Pi_{s}\right)\right)=d\left(0_{n+1}, b d(H)\right) .
$$

Proof. (i) If $\pi \in \Pi_{i} \backslash \Pi_{\infty}$, Proposition 1(iv) ensures that $0_{n} \in \operatorname{cl}(A) \subset \operatorname{cl}\left(Z^{-}\right)$. Moreover, Proposition 2 implies $0_{n+1} \in c l(H)$. Thus,

$$
\begin{aligned}
d\left(0_{n+1}, b d(H)\right) & =d\left(0_{n+1}, \operatorname{ext}(H)\right) \leq d\left(0_{n+1}, \operatorname{ext}(A \times \mathbb{R})\right) \\
& =d\left(0_{n}, \operatorname{ext}(A)\right) \leq d\left(0_{n}, \operatorname{ext}\left(Z^{-}\right)\right) \\
& =d\left(0_{n}, b d\left(Z^{-}\right)\right)
\end{aligned}
$$

(ii) It is immediate as far as $\Pi_{s} \subset \Pi_{c}$, and then if $\pi \in \Pi_{i} \backslash \Pi_{\infty}$, one has, by virtue of Proposition 2(iv),

$$
\delta\left(\pi, b d\left(\Pi_{s}\right)\right) \geq \delta\left(\pi, b d\left(\Pi_{c}\right)\right)=d\left(0_{n+1}, b d(H)\right) .
$$

(iii) From the previous statement it is sufficient to prove that

$$
\delta\left(\pi, b d\left(\Pi_{s}\right)\right) \leq d\left(0_{n+1}, b d(H)\right) .
$$

If $H=\mathbb{R}^{n+1}$, the reader can easily prove that $\pi \in \Pi_{\infty}$, and thus we can take $\left(\begin{array}{l}a \\ b\end{array}\right) \in b d(H)$ such that $d\left(0_{n+1}, b d(H)\right)=\left\|\left(\begin{array}{l}a \\ b\end{array}\right)\right\|$. Consider, then, the problem $\pi_{1}:=$ $\left(c+a, \sigma_{1}\right)$, where

$$
\sigma_{1}:=\left\{\left(a_{t}-a\right)^{\prime} x \geq b_{t}-b ; t \in T\right\} .
$$

Let us see that $\pi_{1} \in b d\left(\Pi_{s}\right)$. We have $\pi_{1} \in b d\left(\Pi_{c}\right)$ (see Proposition 2(iii)). We shall distinguish two possibilities:

(a) In the case when $\pi_{1} \in \Pi_{c}$, Proposition 4 ensures that $\pi_{1} \in b d\left(\Pi_{s}\right)$.

(b) If $\pi_{1} \in \Pi_{i}$, by Proposition $1(\mathrm{v}) 0_{n} \in b d\left(A_{1}\right) \subset \operatorname{cl}\left(Z_{1}^{+}\right)$.

Let us proceed by supposing that, in the latter case, $0_{n} \in \operatorname{int}\left(Z_{1}^{+}\right)$. Since $\pi \in \Pi_{i}$, Proposition 1(iv) gives $0_{n} \in \operatorname{cl}(A) \subset \operatorname{cl}\left(Z^{-}\right)$and, because of the current assumption

$$
\|a\| \leq\left\|\left(\begin{array}{l}
a \\
b
\end{array}\right)\right\|<d\left(0_{n}, b d\left(Z^{-}\right)\right)
$$

it must be the case that $a \in \operatorname{int}\left(Z^{-}\right)$and, then, $0_{n} \in \operatorname{int}\left(Z_{1}^{-}\right)$. Thus, from Lemma 1(iii) we obtain $0_{n} \in \operatorname{int}\left(A_{1}\right)$, which is a contradiction and makes us conclude $0_{n} \in b d\left(Z_{1}^{+}\right)$. Therefore $\pi_{1} \in b d\left(\Pi_{s}\right)$, again by virtue of Proposition 4 . Thus, we have

$$
\delta\left(\pi, b d\left(\Pi_{s}\right)\right) \leq \delta\left(\pi, \pi_{1}\right)=\max \left\{\|a\|,\left\|\left(\begin{array}{l}
a \\
b
\end{array}\right)\right\|\right\}=\left\|\left(\begin{array}{l}
a \\
b
\end{array}\right)\right\| .
$$

Example 4 shows that formula (5) for a problem $\pi \in \Pi_{i} \backslash \Pi_{\infty}$ does not hold in general when $d\left(0_{n+1}, b d(H)\right)=d\left(0_{n}, b d\left(Z^{-}\right)\right)$.

Now we establish an upper bound for the distance to ill-posedness, which coincides with it under certain conditions. We emphasize the fact that this bound works for any $\pi \in \Pi \backslash \Pi_{\infty}$. 
Proposition 9. Let $\pi=(c, \sigma) \in \Pi \backslash \Pi_{\infty}$. Then

$$
\delta\left(\pi, b d\left(\Pi_{s}\right)\right) \leq \max \left\{d\left(0_{n+1}, b d(H)\right),\|c\|\right\} .
$$

Proof. We know that $H \neq \mathbb{R}^{n+1}$ because $\pi \in \Pi \backslash \Pi_{\infty}$. Let $\left(\begin{array}{l}a \\ b\end{array}\right) \in b d(H)$ be such that $d\left(0_{n+1}, b d(H)\right)=\left\|\left(\begin{array}{l}a \\ b\end{array}\right)\right\|$. Consider the problem $\pi_{2}:=\left(0_{n}, \sigma_{2}\right)$, where

$$
\sigma_{2}:=\left\{\left(a_{t}-a\right)^{\prime} x \geq b_{t}-b ; t \in T\right\} .
$$

Let us see that $\pi_{2} \in b d\left(\Pi_{s}\right)$. We have that $\pi_{2} \in b d\left(\Pi_{c}\right)$ (see Proposition 2(iii)). In the case that $\pi_{2} \in \Pi_{c}$, Proposition 4 ensures that $\pi_{2} \in b d\left(\Pi_{s}\right)$. Assuming $\pi_{2} \in$ $\Pi_{i}$, Proposition $1(\mathrm{v})$ ensures $0_{n} \in b d\left(A_{2}\right) \subset \operatorname{cl}\left(Z_{2}^{+}\right)$. If $0_{n} \in \operatorname{int}\left(Z_{2}^{+}\right)$, the second statement in Proposition 7 entails $0_{n} \in \operatorname{int}\left(A_{2}\right)$, which is a contradiction. Thus, $0_{n} \in b d\left(Z_{2}^{+}\right)$, and then $\pi_{2} \in b d\left(\Pi_{s}\right)$, again by Proposition 4 . Thus we have

$$
\delta\left(\pi, b d\left(\Pi_{s}\right)\right) \leq \delta\left(\pi, \pi_{2}\right)=\max \left\{d\left(0_{n+1}, b d(H)\right),\|c\|\right\} .
$$

The following result can be obtained as a straightforward consequence of the previous statements and establishes bounds on the distance to ill-posedness.

Theorem 5. Let $\pi=(c, \sigma) \in \Pi \backslash \Pi_{\infty}$. If we denote

$$
\begin{aligned}
\alpha & :=\min \left\{d\left(0_{n+1}, b d(H)\right), d\left(0_{n}, b d\left(Z^{-}\right)\right)\right\} \text {and } \\
\beta & :=\max \left\{d\left(0_{n+1}, b d(H)\right),\|c\|\right\},
\end{aligned}
$$

then we have

$$
\alpha \leq \delta\left(\pi, b d\left(\Pi_{s}\right)\right) \leq \beta .
$$

Figure 2 synthesizes the main results about the distance to ill-posedness with respect to the solvability provided in this paper. The reader should analyze the information provided in this figure together with that given in Figure 1. We use the same notation as in Theorem 5 for $\alpha$ and $\beta$.

4. Other upper bounds. The goal of the following result is to obtain, under additional hypotheses, some refinements of the upper bound given in Proposition 9.

Proposition 10. Let $\pi=(c, \sigma) \in \operatorname{ext}\left(\Pi_{s}\right)$. The following statements hold:

(i) If $\pi \in \operatorname{cl}\left(\Pi_{c}\right)$, then

$$
\delta\left(\pi, b d\left(\Pi_{s}\right)\right) \leq d(c, b d(M)) \leq\|c\| .
$$

(ii) If $\pi \in b d\left(\Pi_{c}\right)$, then

$$
\delta\left(\pi, b d\left(\Pi_{s}\right)\right) \leq d(c, b d(-M)) \leq d(c, b d(M)) .
$$

(iii) If $\pi \in b d\left(\Pi_{c}\right)$ and there exists $t_{0} \in T$ such that $a_{t_{0}}=0_{n}$, then

$$
\delta\left(\pi, b d\left(\Pi_{s}\right)\right) \leq d\left(0_{n}, b d\left(Z^{+}\right)\right) \leq d(c, b d(-M)) .
$$

Proof. (i) First observe that $M \neq \mathbb{R}^{n}$ since, otherwise, either $\pi \in \operatorname{cl}\left(\Pi_{s}\right)$ (when $\pi \in \Pi_{c}$ and applying Proposition 1(iii)) or $\pi \in \operatorname{int}\left(\Pi_{i}\right)$ (when $\pi \in \Pi_{i}$ and applying Proposition 1(vi)).

Let $a \in b d(M)$ such that $d(c, b d(M))=\|c-a\|$ and consider the problem $\pi_{3}:=$ $(a, \sigma)$. We have $\pi_{3} \in c l\left(\Pi_{c}\right)$ and $a \in b d\left(M_{3}\right)=b d(M)$. If $\pi_{3} \in \Pi_{c}$, Proposition 1(iii) 


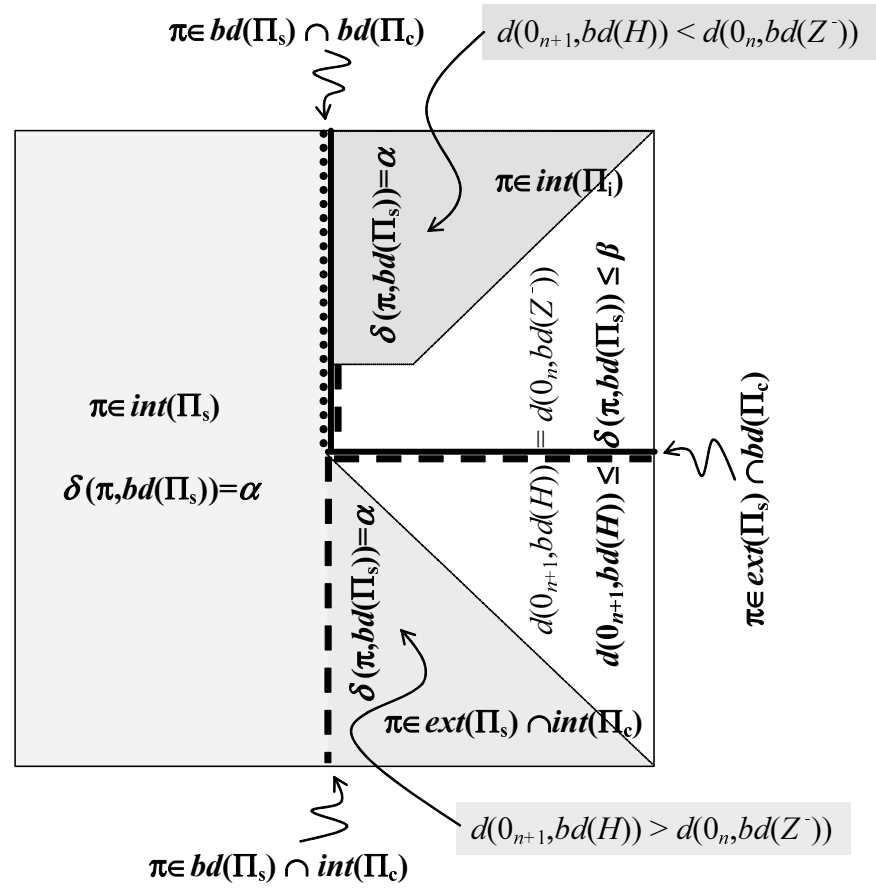

FIG. 2. Distance to ill-posedness in $\Pi \backslash \Pi_{\infty}$.

ensures that $\pi_{3} \in \operatorname{cl}\left(\Pi_{s}\right)$. If $\pi_{3} \in b d\left(\Pi_{c}\right)$, then $\pi_{3} \in b d\left(\Pi_{s}\right)$ by Lemma 1(ii). In any case, $\pi_{3} \in \operatorname{cl}\left(\Pi_{s}\right)$, and then

$$
\delta\left(\pi, b d\left(\Pi_{s}\right)\right) \leq \delta\left(\pi, \pi_{3}\right)=\|c-a\|=d(c, b d(M)) .
$$

Finally, and since $M \neq \mathbb{R}^{n}$, one has $0_{n} \in b d(M)$, which implies

$$
d(c, b d(M)) \leq\|c\| .
$$

(ii) Obviously we have again $M \neq \mathbb{R}^{n}$. Let $d \in b d(-M)$ satisfying that $d(c, b d(-M))$ $=\|c-d\|$ and consider the problem $\pi_{4}:=(d, \sigma)$. Obviously $\pi_{4} \in b d\left(\Pi_{c}\right)$ and we see that $\pi_{4} \in b d\left(\Pi_{s}\right)$. In the case when $\pi_{4} \in \Pi_{c}$, Proposition 4 ensures $\pi_{4} \in b d\left(\Pi_{s}\right)$. If $\pi_{4} \in \Pi_{i}$, from Proposition $1(\mathrm{v})$ one has $0_{n} \in b d\left(A_{4}\right) \subset \operatorname{cl}\left(Z_{4}^{+}\right)$.

If $0_{n} \in \operatorname{int}\left(Z_{4}^{+}\right)$, then $-d \in \operatorname{int}\left(M_{4}\right)=\operatorname{int}(M)$ (see Proposition 7), i.e., $d \in$ int $(-M)$, which is a contradiction. Then $0_{n} \in b d\left(Z_{4}^{+}\right)$and, again by Proposition 4, $\pi_{4} \in b d\left(\Pi_{s}\right)$. So, in any case, $\pi_{4} \in b d\left(\Pi_{s}\right)$, and then

$$
\delta\left(\pi, b d\left(\Pi_{s}\right)\right) \leq \delta\left(\pi, \pi_{4}\right)=\|c-d\|=d(c, b d(-M)) .
$$

Because of Proposition 1(iv) we have $0_{n} \in \operatorname{cl}(A) \subset \operatorname{cl}\left(Z^{+}\right)$, and $0_{n} \in b d\left(Z^{+}\right)$ leads us to the contradiction $\pi \in b d\left(\Pi_{s}\right)$ (by Proposition 4$)$. Hence we have that, under the current hypothesis, $0_{n} \in \operatorname{int}\left(Z^{+}\right)$or, equivalently, $-c \in \operatorname{int}(M) \Leftrightarrow c \in \operatorname{int}(-M)$. Since $M \neq \mathbb{R}^{n}$, there will exist $u \neq 0_{n}$ such that $M$ and $-M$ are, respectively, contained in the half-spaces $S_{+}:=\left\{x \in \mathbb{R}^{n} \mid u^{\prime} x \leq 0\right\}$ and $S_{-}:=\left\{x \in \mathbb{R}^{n} \mid u^{\prime} x \geq 0\right\}$. Therefore

$$
d(c, b d(-M)) \leq d\left(c, S_{+}\right) \leq d(c, b d(M)) .
$$


(iii) Remember that under the current hypotheses $0_{n} \in \operatorname{int}\left(Z^{+}\right)$. Take $a \in$ $b d\left(Z^{+}\right)$such that $d\left(0_{n}, b d\left(Z^{+}\right)\right)=\|a\|$. Since $a \in b d\left(Z^{+}\right)$, one has $0_{n} \in b d\left(Z^{+}-a\right)$.

Consider the problem $\pi_{5}:=\left(c-a, \sigma_{5}\right)$ where $\sigma_{5}:=\left\{\left(a_{t}^{5}\right)^{\prime} x \geq b_{t} ; t \in T\right\}$ with $a_{t}^{5}:=a_{t}-a$ if $t \in T \backslash\left\{t_{0}\right\}$ and $a_{t_{0}}^{5}:=a_{t_{0}}=0_{n}$. From the definition of $\pi_{5}$ one has, obviously, $0_{n} \in \operatorname{cl}\left(Z_{5}^{+}\right)$. If we had $0_{n} \in \operatorname{int}\left(Z_{5}^{+}\right)$, then we would obtain the following contradiction:

$$
0_{n} \in \operatorname{int}\left(\operatorname{conv}\left(\left\{a_{t}-a, t \in T \backslash\left\{t_{0}\right\} ; c-a\right\}\right)\right) \subset \operatorname{int}\left(Z^{+}-a\right) .
$$

Then $0_{n} \in b d\left(Z_{5}^{+}\right)$and, in particular, $0_{n+1} \notin \operatorname{int}\left(H_{5}\right)$ (otherwise $0_{n} \in \operatorname{int}\left(A_{5}\right) \subset$ $\left.\operatorname{int}\left(Z_{5}^{+}\right)\right)$. Thus, Proposition 2 yields $\pi_{5} \in \operatorname{cl}\left(\Pi_{c}\right)$ and we have the following discussion. If $\pi_{5} \in b d\left(\Pi_{c}\right)$, then Proposition 4 leads us to conclude that $\pi_{5} \in b d\left(\Pi_{s}\right)$. If $\pi_{5} \in \operatorname{int}\left(\Pi_{c}\right)$, since $0_{n} \in A_{5} \subset \operatorname{cl}\left(Z_{5}^{-}\right)$, Proposition 3 entails $\pi_{5} \in c l\left(\Pi_{s}\right)$. In any case, we have $\pi_{5} \in \operatorname{cl}\left(\Pi_{s}\right)$, and then

$$
\delta\left(\pi, b d\left(\Pi_{s}\right)\right) \leq \delta\left(\pi, \pi_{5}\right)=\|a\|=d\left(0_{n}, b d\left(Z^{+}\right)\right) .
$$

Remember that under the current hypotheses we have $-c \in \operatorname{int}(M)$, i.e., $0_{n} \in$ $\operatorname{int}(M+c)$, and the latter implies $\mu(M+c) \subset M+c$ for every $0 \leq \mu \leq 1$, due to the convexity of $M+c$. Now we will prove that $Z^{+} \subset M+c$. In fact, if $x \in Z^{+}$there exist $a \in A$ and $\lambda \geq 0, \mu \geq 0$, with $\lambda+\mu=1$, such that $x=\lambda a+\mu c$. If $\mu>0$, we can write

$$
x=\mu\left(\frac{\lambda}{\mu} a+c\right) \in \mu(M+c) \subset M+c,
$$

and if $\mu=0$, we have $x=a \in A \subset M \subset M+c$, where the latter inclusion is due to the fact that $-c \in M$. Thus, we have

$$
\begin{aligned}
d\left(0_{n}, b d\left(Z^{+}\right)\right) & \leq d\left(0_{n}, b d(M+c)\right)=d\left(0_{n}, b d(M)+c\right) \\
& =d(-c, b d(M))=d(c, b d(-M)) .
\end{aligned}
$$

5. Examples and counterexamples. In this section we present different examples in order to show the usefulness of formula (5) for obtaining the distance to ill-posedness for problems satisfying the hypothesis of Theorem 1. Moreover, some of these examples show that this formula does not generally provide the desired distance when the hypotheses of Theorem 1 are not fulfilled. Different situations illustrate that certain redundant constraints may considerably affect the distance to ill-posedness, even for ordinary linear programming problems with "few" constraints. In fact, we provide two linear optimization problems in $\mathbb{R}^{2}$ (Examples 5 and 6) with the same associated sets $A, M, Z^{-}, Z^{+}, C$, and $H$, and different distances to ill-posedness. These examples show that the sets $A, M, Z^{-}, Z^{+}, C$, and $H$ alone are not sufficient to characterize the distance to ill-posedness in all cases. The norm considered (in $\mathbb{R}^{n}$ and $\mathbb{R}^{n+1}$ ) in all the examples is $\|\cdot\|_{\infty}$.

The following examples illustrate formula (5) of Theorem 2 for the problem $\pi \in$ $c l\left(\Pi_{s}\right)$.

Example 1. Consider the linear optimization problem in $\mathbb{R}^{2}$,

$$
\begin{array}{rr}
\pi: & \text { Inf } \\
& \frac{1}{2} x_{2} \\
\text { s.t. } & -x_{1}+x_{2} \geq 0, \\
& x_{1}+x_{2} \geq 0 .
\end{array}
$$


One has

$$
H=\operatorname{conv}\left\{(-1,1,0)^{\prime},(1,1,0)^{\prime}\right\}+\mathbb{R}_{+}\left\{(0,0,-1)^{\prime}\right\}
$$

and

$$
Z^{-}=\operatorname{conv}\left\{(-1,1)^{\prime},(1,1)^{\prime},(0,-1 / 2)^{\prime}\right\} .
$$

Since $0_{3} \in \operatorname{ext}(H)$, then $\pi \in \operatorname{int}\left(\Pi_{c}\right)$ (Proposition 2(iii)) and then, since $0_{2} \in$ $\operatorname{int}\left(Z^{-}\right)$, one obtains $\pi \in \operatorname{int}\left(\Pi_{s}\right)$ (Proposition 3(iii)). It is easy to check that

$$
\frac{1}{5}=\left\|\left(\begin{array}{c}
\frac{1}{5} \\
\frac{-1}{5}
\end{array}\right)\right\|_{\infty}=d\left(0_{2}, b d\left(Z^{-}\right)\right)<d\left(0_{3}, b d(H)\right)=1 .
$$

Then, $\delta\left(\pi, b d\left(\Pi_{s}\right)\right)=d\left(0_{2}, b d\left(Z^{-}\right)\right)=1 / 5$.

As in the proof of Theorem 2, a problem belonging to $b d\left(\Pi_{s}\right)$, where this distance is attained, is given by

$$
\begin{aligned}
\pi_{0}: & \operatorname{Inf} \\
& \left(0+\frac{1}{5}\right) x_{1}+\left(\frac{1}{2}-\frac{1}{5}\right) x_{2} \\
\text { s.t. } \quad\left(-1-\frac{1}{5}\right) x_{1}+\left(1+\frac{1}{5}\right) x_{2} & \geq 0-0, \\
& \left(1-\frac{1}{5}\right) x_{1}+\left(1+\frac{1}{5}\right) x_{2} \geq 0-0 .
\end{aligned}
$$

We have $0_{2} \in \operatorname{bd}\left(Z_{0}^{-}\right)$and $0_{3} \in \operatorname{ext}\left(H_{0}\right)$. Consequently $\pi_{0} \in \operatorname{int}\left(\Pi_{c}\right) \cap b d\left(\Pi_{s}\right)$ (Propositions 2(ii) and 3(ii)).

Example 2. Consider now the linear optimization problem in $\mathbb{R}^{2}$,

$$
\pi: \begin{aligned}
\text { Inf } \quad 2 x_{2} & \\
\text { s.t. }-x_{1}+x_{2} & \geq 0, \\
& x_{1}+x_{2} \geq 0 \\
& \geq \frac{1}{4} x_{2} \geq 0 .
\end{aligned}
$$

Now we have

$$
H=\operatorname{conv}\left\{(-1,1,0)^{\prime},(1,1,0)^{\prime},(0,1 / 4,0)^{\prime}\right\}+\mathbb{R}_{+}\left\{(0,0,-1)^{\prime}\right\}
$$

and

$$
Z^{-}=\operatorname{conv}\left\{(-1,1)^{\prime},(1,1)^{\prime},(0,1 / 4)^{\prime},(0,-2)^{\prime}\right\} .
$$

As in the previous example one has $\pi \in \operatorname{int}\left(\Pi_{s}\right)$. Again it is easy to check that

$$
\frac{1}{2}=d\left(0_{2}, b d\left(Z^{-}\right)\right)>d\left(0_{3}, b d(H)\right)=\left\|\left(0, \frac{1}{4}, 0\right)^{\prime}\right\|_{\infty}=\frac{1}{4},
$$

and $\delta\left(\pi, b d\left(\Pi_{s}\right)\right)=d\left(0_{3}, b d(H)\right)=1 / 4$.

Now, the problem $\pi_{0} \in b d\left(\Pi_{s}\right)$ where this distance is attained, is given by

$$
\begin{aligned}
\pi_{0}: \quad \begin{array}{l}
\text { Inf } \quad 2 x_{2} \\
\text { s.t. } \quad(-1-0) x_{1}+\left(1-\frac{1}{4}\right) x_{2}
\end{array} & \geq 0-0, \\
(1-0) x_{1}+\left(1-\frac{1}{4}\right) x_{2} & \geq 0-0, \\
& (0-0) x_{1}+\left(\frac{1}{4}-\frac{1}{4}\right) x_{2} \geq 0-0 .
\end{aligned}
$$


We have $0_{3} \in b d\left(H_{0}\right)$. In fact $0_{3} \in b d\left(C_{0}\right)$, and then we conclude that $\pi_{0} \in b d\left(\Pi_{s}\right)$ (by virtue of Proposition 6(ii)).

Examples 3 and 4 (see also Example 5 ) show that formula (5) of Theorem 1 cannot be extended to the case $\pi \in \operatorname{ext}\left(\Pi_{s}\right)$ when $d\left(0_{n}, b d\left(Z^{-}\right)\right)=d\left(0_{n+1}, b d(H)\right)<\|c\|$, not even in the context of ordinary linear programming in $\mathbb{R}$. The characterization of $b d\left(\Pi_{s}\right)$ given in Proposition 6 is used in the remaining examples.

Example 3. Consider now the ordinary linear programming problem in $\mathbb{R}$,

$$
\begin{array}{ll}
\pi: & \text { Inf } \quad-10 x \\
& \text { s.t. } \quad x \geq 9, \quad x \geq 10, \quad 4 x \geq 9, \quad 4 x \geq 10 .
\end{array}
$$

One has that $\pi \in \operatorname{int}\left(\Pi_{c}\right) \cap \operatorname{ext}\left(\Pi_{s}\right)$ and $d\left(0, b d\left(Z^{-}\right)\right)=1=d\left(0_{2}, b d(H)\right)$. Moreover, $\delta\left(\pi, b d\left(\Pi_{s}\right)\right)=4>d\left(0_{2}, b d(H)\right)$, and this is attained in the problem

$$
\begin{aligned}
\pi_{1}: & \text { Inf } \quad-10 x \\
& \text { s.t. } \quad-3 x \geq 9, \quad-3 x \geq 10, \quad 0 x \geq 9, \quad 0 x \geq 10 .
\end{aligned}
$$

Indeed, if $\pi_{2} \in \Pi$ is such that $\delta\left(\pi, \pi_{2}\right)<4$, Proposition 8 guarantees that $0 \in \operatorname{int}\left(Z_{2}^{+}\right)$ and $0_{2} \in \operatorname{ext}\left(C_{2}\right)$. The coefficients of the problem imply that $0_{2} \in \operatorname{ext}\left(H_{2}\right)$ only if $\left.H_{2} \subset\right] 0,+\infty\left[\times \mathbb{R}\right.$, in which case one also has $0_{2} \in \operatorname{ext}\left(Z_{2}^{-}\right)$. So it is impossible to have $\pi_{2} \in b d\left(\Pi_{s}\right)$ according to Proposition 6 .

Observe that, in this case, the problems of $b d\left(\Pi_{s}\right)$ at which the distance $\delta\left(\pi, b d\left(\Pi_{s}\right)\right)$ is attained verify the third condition in Proposition 6 .

Example 4. Consider the linear programming problem in $\mathbb{R}$,

$$
\begin{array}{lll}
\pi: & \text { Inf } & -3 x \\
& \text { s.t. } & -x \geq 5, \quad 2 x \geq 5, \quad-x \geq 4, \quad 2 x \geq 4 .
\end{array}
$$

One has that $\pi \in \operatorname{int}\left(\Pi_{i}\right)$ and $d\left(0_{2}, b d(H)\right)=d\left(0, b d\left(Z^{-}\right)\right)=1$. It is easy to check that

$$
\delta\left(\pi, b d\left(\Pi_{s}\right)\right)=\delta\left(\pi, \pi_{1}\right)=2>d\left(0_{2}, b d(H)\right),
$$

where

$$
\begin{aligned}
& \pi_{1}: \text { Inf }-3 x \\
& \text { s.t. } \quad-3 x \geq 5, \quad 0 x \geq 5, \quad-3 x \geq 4, \quad 0 x \geq 4 \text {. }
\end{aligned}
$$

In the following examples, the considered problems have the same sets $A, M$, $Z^{-}, Z^{+}, C$, and $H$, but their distances to ill-posedness are different. Moreover, in the second example the distance to ill-posedness obeys formula (5), although the problem does not satisfy the hypotheses of Theorem 1.

Example 5. Consider the problem

$$
\begin{aligned}
\pi: \text { Inf } \quad 6 x_{1}-3 x_{2} & \\
\text { s.t. } \quad 3 x_{1}+x_{2} & \geq 10, \quad t=1, \\
3 x_{1}+3 x_{2} & \geq 10, \quad t=2, \\
-2 x_{1}+3 x_{2} & \geq 10, \quad t=3, \\
-2 x_{1}+x_{2} & \geq 10, \quad t=4 .
\end{aligned}
$$

It is easy to check that $\pi \in \operatorname{int}\left(\Pi_{c}\right) \cap \operatorname{ext}\left(\Pi_{s}\right)$ and $d\left(0_{2}, b d\left(Z^{-}\right)\right)=1=d\left(0_{3}, b d(H)\right)$. Now let us see that $\delta\left(\pi, b d\left(\Pi_{s}\right)\right)>1$. The choice of $b_{t}=10, t=1, \ldots, 4$, allows us 


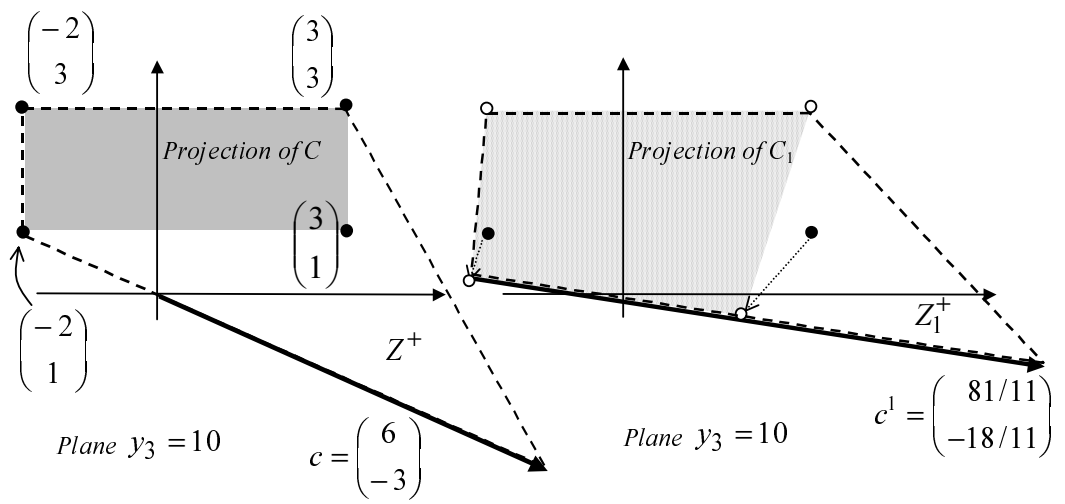

FIG. 3. Perturbation of $\pi$ to obtain condition (iii) of Proposition 6.

to see that the nearest problem to $\pi, \pi_{1} \in b d\left(\Pi_{s}\right)$ does not verify the condition $0_{3} \in b d\left(C_{1}\right)$. Thus, we will look for a problem $\pi_{1}=\left(c^{1}, \sigma_{1}\right)$, with $\sigma_{1}=\left\{\left(a_{t}^{1}\right)^{\prime} x \geq\right.$ $\left.b_{t}^{1}, t=1, \ldots, 4\right\}$, as the one presented in Figure 3 below, where we illustrate graphically the sets $C$ and $Z^{+}$, associated with $\pi$, as well as the ones associated with $\pi_{1}$. It can be proved that the minimum perturbation (in $\|\cdot\|_{\infty}$ ) for which $c^{1}$ is a multiple of $a_{1}^{1}$ corresponds to the vectors $c^{1}=\left(\begin{array}{c}81 / 11 \\ -18 / 11\end{array}\right)$ and $a_{1}^{1}=\left(\begin{array}{c}18 / 11 \\ -4 / 11\end{array}\right)$, both colinear with $\left(\begin{array}{c}9 \\ -2\end{array}\right)$. Now we modify $a_{4}$ to get $0_{3} \in b d\left(H_{1}\right)$ and $0_{2} \in b d\left(Z_{1}^{+}\right)$, taking for example $a_{4}^{1}=\left(\begin{array}{c}-27 / 11 \\ 6 / 11\end{array}\right)$. The remaining coefficients stay unchanged. For simplicity, and since $b_{t}^{1}=b_{t}=10$, for $t=1, \ldots, 4$, we represent the figures projected in the plane $\left\{y \in \mathbb{R}^{3} \mid y_{3}=10\right\}$.

Next we will check that if $\pi_{2}=\left(c^{2}, \sigma_{2}\right) \in \Pi$ verifies $\delta\left(\pi_{2}, \pi\right)<\delta\left(\pi_{1}, \pi\right)=\frac{15}{11}$, then $\pi_{2} \notin \Pi_{s}$. Indeed, in other case and appealing to Proposition 1(ii), we will obtain $c^{2} \in M_{2}$ (which is a closed cone because it is finitely generated). Moreover, one can easily check that the following inequalities relative to $\sigma_{2}=\left\{\left(a_{t}^{2}\right)^{\prime} x \geq b_{t}^{2}, t=1, \ldots, 4\right\}$ hold:

$$
\begin{aligned}
\left(a_{1}^{2}\right)^{\prime}\left(\begin{array}{c}
9 \\
-2
\end{array}\right)>0,\left(a_{2}^{2}\right)^{\prime}\left(\begin{array}{c}
9 \\
-2
\end{array}\right)>0,\left(a_{3}^{2}\right)^{\prime}\left(\begin{array}{c}
9 \\
-2
\end{array}\right)<0,\left(a_{4}^{2}\right)^{\prime}\left(\begin{array}{c}
9 \\
-2
\end{array}\right)<0, \\
\left(a_{1}^{2}\right)^{\prime}\left(\begin{array}{c}
2 \\
9
\end{array}\right)>0, \quad\left(a_{2}^{2}\right)^{\prime}\left(\begin{array}{l}
2 \\
9
\end{array}\right)>0, \quad\left(a_{3}^{2}\right)^{\prime}\left(\begin{array}{c}
2 \\
9
\end{array}\right)>0, \\
\left(c^{2}\right)^{\prime}\left(\begin{array}{c}
9 \\
-2
\end{array}\right)>0 \\
\left(c^{2}\right)^{\prime}\left(\begin{array}{l}
2 \\
9
\end{array}\right)<0 .
\end{aligned}
$$

The condition $c^{2} \in M_{2}$, together with (7) and (9), implies that $\left(a_{4}^{2}\right)^{\prime}\left(\begin{array}{l}2 \\ 9\end{array}\right)<0$; then the vectors $a_{1}^{2}, a_{3}^{2}, a_{4}^{2}$, and $c^{2}$ are, respectively, in the interior of the first second, third, and fourth quadrants determined by the (orthogonal) vectors $\left(\begin{array}{c}9 \\ -2\end{array}\right)$ and $\left(\begin{array}{l}2 \\ 9\end{array}\right)$, from where one deduces that $M_{2}=\mathbb{R}^{2}$. That is, $0_{2} \in \operatorname{int}\left(\operatorname{conv}\left(\left\{a_{t}^{2}, t=1, \ldots, 4\right\}\right)\right)$, and since $b_{t}^{2} \geq 10-\frac{15}{11}>0$ for all $t=1, \ldots, 4$, we conclude that $0_{3} \in \operatorname{int}\left(H_{2}\right)$, and then $\pi_{2} \in \operatorname{int}\left(\Pi_{i}\right)$ (Proposition 2(i)), in contradiction with the assumption $\pi_{2} \in \Pi_{s}$. Thus we conclude that $\delta\left(\pi, b d\left(\Pi_{s}\right)\right)=\delta\left(\pi, \pi_{1}\right)=\frac{15}{11}$.

Example 6. Consider the linear programming problem $\pi$ in $\mathbb{R}^{2}$, obtained by adding to the problem of the previous example the constraint $0 x_{1}+x_{2} \geq 10$ (for $t=5$ ), whose coefficient vector is a convex combination of the vectors associated with 


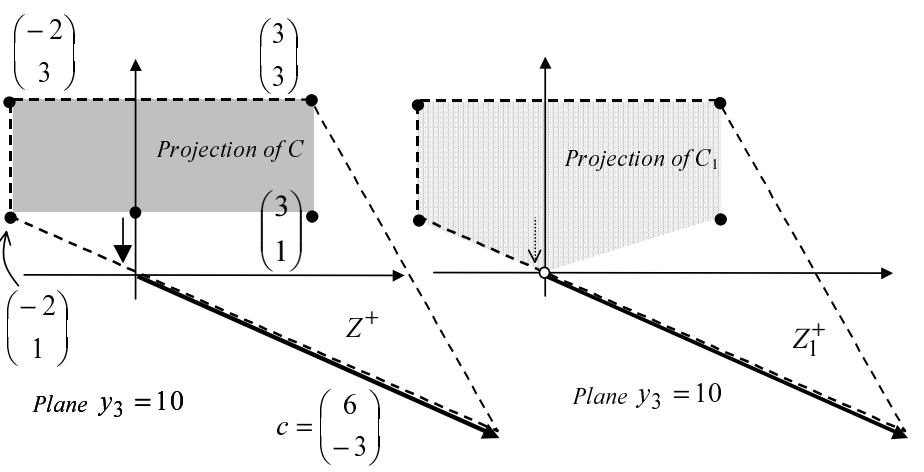

FIG. 4. Perturbation of $\pi$ to obtain condition (iii) of Proposition 6.

the first and fourth constraints (in particular, the new constraint is redundant). This problem still verifies the conditions $\pi \in \operatorname{int}\left(\Pi_{c}\right) \cap \operatorname{ext}\left(\Pi_{s}\right)$ and $d\left(0_{2}, b d\left(Z^{-}\right)\right)=1=$ $d\left(0_{3}, b d(H)\right)$. However, now one has $\delta\left(\pi, b d\left(\Pi_{s}\right)\right)=d\left(0_{3}, b d(H)\right)$, since $d\left(0_{3}, b d(H)\right)$ is a lower bound for the distance from $\pi$ to $b d\left(\Pi_{s}\right)$ (see Theorem 3 ) and the problem $\pi_{1}$, coming from replacing the last constraint of $\pi$ by the new one " $0 x_{1}+0 x_{2} \geq 10$," verifies that $\pi_{1} \in b d\left(\Pi_{s}\right)$ (since $0_{n+1} \in b d\left(H_{1}\right)$ and $\left.0_{n} \in b d\left(Z_{1}^{+}\right)\right)$and $\delta\left(\pi, \pi_{1}\right)=1$. In Figure 4 we illustrate graphically these elements, projecting them on the plane $y_{3}=10$ (note that $b_{t}=10$, for all $\left.t=1, \ldots, 4\right)$.

6. A primal-dual approach to the distance to solvability/unsolvability. Let us consider the dual of problem (1), which is given by

$$
\begin{gathered}
\pi^{d}: \quad \operatorname{Sup} \sum_{t \in T} \lambda_{t} b_{t} \\
\text { s.t. } \sum_{t \in T} \lambda_{t} a_{t}=c, \\
\lambda \in \mathbb{R}_{+}^{(T)},
\end{gathered}
$$

where $\mathbb{R}_{+}^{(T)}$ is the convex cone of all the functions $\lambda: T \rightarrow \mathbb{R}_{+}$taking positive values only at finitely many points of $T$. When $T$ is infinite, $\pi^{d}$ is also a linear semi-infinite programming problem having, in this case a finite number of constraints but an infinite number of variables. In this case, $\pi^{d}$ is called the dual of $\pi$ in the sense of Haar. The subset of $\Pi$ formed by those problems whose dual is consistent will be denoted by $\Pi_{c}^{d}$; in other words, $\Pi_{c}^{d}:=\{\pi \in \Pi \mid c \in M\}$.

In the finite case ( $T$ finite) it is well known from linear programming duality that the problem $\pi$ is solvable if and only if it is both primal and dual feasible; i.e., $\Pi_{s}=\Pi_{c} \cap \Pi_{c}^{d}$. Hence, for solvable instances the distance to unsolvability is given by

$$
\delta\left(\pi, b d\left(\Pi_{s}\right)\right)=\min \left\{\delta\left(\pi, b d\left(\Pi_{c}\right)\right), \delta\left(\pi, b d\left(\Pi_{c}^{d}\right)\right)\right\} .
$$

The finite case is actually a particular case of the conic linear context studied in [5], [10], [16], [18], [19], and [20], among others. In section 7, which is specifically devoted to the finite case, we show how some results of [10], [19], and [20] may be used to derive, for $\pi \in \Pi_{s}$, the expressions

$$
\delta\left(\pi, b d\left(\Pi_{c}\right)\right)=d\left(0_{n+1}, b d(H)\right) \text { and } \delta\left(\pi, b d\left(\Pi_{c}^{d}\right)\right)=d\left(0_{n}, b d\left(Z^{-}\right)\right) .
$$


The first equality is a particular case of Proposition 2(iv), and the second is extended, in Theorem 6, to the semi-infinite case and for problems without any consistency requirements.

First of all, let us point out that when $T$ is infinite, solvability may not be identified with primal-dual feasibility. In fact, none of the inclusions $\Pi_{s} \supset \Pi_{c} \cap \Pi_{c}^{d}$ and $\Pi_{s} \subset$ $\Pi_{c} \cap \Pi_{c}^{d}$ holds, as the following examples show. Despite this fact, Corollary 1 will show that (11) remains valid for problems in $c l\left(\Pi_{s}\right)$ as well as for some subset of insolvable problems.

Example $7 . \Pi_{s} \nsupseteq \Pi_{c} \cap \Pi_{c}^{d}$. Let us consider the linear semi-infinite programming problem in $\mathbb{R}^{2}$,

$$
\begin{aligned}
& \pi: \quad \operatorname{Inf} x_{1} \\
& \text { s.t. } \left.t x_{1}+\frac{1}{t} x_{2} \geq 2, t \in\right] 0,+\infty[\text {, } \\
& x_{1} \geq 0 \text {. }
\end{aligned}
$$

One can easily check that $F=\left\{\left(x_{1}, x_{2}\right)^{\prime} \mid x_{2} \geq x_{1}^{-1}, x_{1}>0\right\}$ and then $\pi$ is bounded but not solvable. However, $\pi \in \Pi_{c} \cap \Pi_{c}^{d}$.

Example 8. $\Pi_{s} \nsubseteq \Pi_{c} \cap \Pi_{c}^{d}$. Let us consider the problem in $\mathbb{R}^{2}$,

$$
\begin{array}{ll}
\pi: & \operatorname{Inf} x_{1} \\
& \text { s.t. } \left.\quad x_{1}+t x_{2} \geq 0, t \in\right] 0,+\infty[.
\end{array}
$$

The feasible set of $\pi$ coincides with $\left[0,+\infty\left[\left[^{2}\right.\right.\right.$ and then it is immediate that $\pi \in \Pi_{s}$. However, $M=] 0,+\infty\left[^{2} \cup\left\{0_{2}\right\}\right.$ and then $c=(1,0)^{\prime} \notin M$, i.e., $\pi \notin \Pi_{c}^{d}$.

The following theorem is a dual version of Proposition 2. It characterizes the illposedness with respect to the dual consistency and provides the associated distance to ill-posedness.

TheOREM 6. Let $\pi \in \Pi$. The following statements hold:

(i) $\pi \in \operatorname{int}\left(\Pi_{c}^{d}\right)$ if and only if $0_{n} \in \operatorname{int}\left(Z^{-}\right)$;

(ii) $\pi \in b d\left(\Pi_{c}^{d}\right)$ if and only if $0_{n} \in b d\left(Z^{-}\right)$;

(iii) $\pi \in \operatorname{ext}\left(\Pi_{c}^{d}\right)$ if and only if $0_{n} \in \operatorname{ext}\left(Z^{-}\right)$;

(iv) $\delta\left(\pi, b d\left(\Pi_{c}^{d}\right)\right)=d\left(0_{n}, b d\left(Z^{-}\right)\right)$.

Proof. (i) Theorem 5 in [14] establishes that $\pi \in \operatorname{int}\left(\Pi_{c}^{d}\right)$ if and only if $c \in \operatorname{int}(M)$, which is equivalent to $0_{n} \in \operatorname{int}\left(Z^{-}\right)$(see Proposition 7).

(ii) Let us start with the "only if" part. Take $\pi \in b d\left(\Pi_{c}^{d}\right)$ and a sequence $\left\{\pi_{r}\right\} \subset \Pi_{c}^{d}$ converging to $\pi$. We have, on the one hand, $c^{r} \in M_{r}$ for each $r \in \mathbb{N}$ and consequently $0_{n} \in Z_{r}^{-}$. On the other hand, $0_{n} \notin \operatorname{int}\left(Z^{-}\right)$because $\pi \in b d\left(\Pi_{c}^{d}\right)$. Assume by contradiction that $0_{n} \notin b d\left(Z^{-}\right)$. Then $0_{n} \in \operatorname{ext}\left(Z^{-}\right)$and, since $\left\{\pi_{r}\right\}$ converges to $\pi$, Proposition 8 ensures that $0_{n} \in \operatorname{ext}\left(Z_{r}^{-}\right)$for $r$ large enough, which represents a contradiction. Therefore, $0_{n} \in b d\left(Z^{-}\right)$.

In order to prove the "if" condition, assume that $0_{n} \in b d\left(Z^{-}\right)$and take a sequence $\left\{u^{r}\right\} \subset Z^{-}$converging to $0_{n}$. We can write

$$
u^{r}=\sum_{t \in T} \lambda_{t}^{r} a_{t}-\mu_{r} c, \quad r=1,2, \ldots
$$

for some sequences $\left\{\lambda^{r}\right\} \subset \mathbb{R}_{+}^{(T)}$ and $\left\{\mu_{r}\right\} \subset \mathbb{R}_{+}$verifying $\sum_{t \in T} \lambda_{t}^{r}+\mu_{r}=1$. For each $r \in \mathbb{N}$, define the problem $\pi_{r}:=\left(c^{r}, \sigma_{r}\right)$ by distinguishing two cases: 
(1) If $\mu_{r}=0$, let $c^{r}:=c$ and

$$
\sigma_{r}:=\left\{\left(a_{t}-u^{r}+\frac{1}{r} c\right)^{\prime} x \geq b_{t}, t \in T\right\} .
$$

Note that in this case $\sum_{t \in T} \lambda_{t}^{r}=1$ and $\sum_{t \in T} \lambda_{t}^{r}\left(a_{t}-u^{r}+\frac{1}{r} c\right)=\frac{1}{r} c$, so $c^{r}=c \in M_{r}$.

(2) If $\mu_{r}>0$, defining $c^{r}:=c+u^{r}$ and

$$
\sigma_{r}:=\left\{\left(a_{t}-u^{r}\right)^{\prime} x \geq b_{t}, t \in T\right\},
$$

one has $\sum_{t \in T} \lambda_{t}^{r}\left(a_{t}-u^{r}\right)=\mu_{r}\left(c+u^{r}\right)$ and, again, $c^{r} \in M_{r}$.

Thus, in any case, $\pi_{r} \in \Pi_{c}^{d}$ and, since $\left\{\pi_{r}\right\}$ converges to $\pi$, then $\pi \in \operatorname{cl}\left(\Pi_{c}^{d}\right)$. Finally, under the current hypothesis, $\pi \notin \operatorname{int}\left(\Pi_{c}^{d}\right)$ and then $\pi \in b d\left(\Pi_{c}^{d}\right)$.

(iii) This is a straightforward consequence of (i) and (ii).

(iv) If $\pi \in b d\left(\Pi_{c}^{d}\right)$, the desired equality comes trivially from (ii). Assume that $\pi \in \operatorname{int}\left(\Pi_{c}^{d}\right)$, and let us see first the inequality " $\geq$." If $\pi_{1} \in \Pi$ satisfies $\delta\left(\pi, \pi_{1}\right)<$ $d\left(0_{n}, b d\left(Z^{-}\right)\right)$, then Proposition 8 implies $0_{n} \in \operatorname{int}\left(Z_{1}^{-}\right)$and thus $\pi_{1} \in \operatorname{int}\left(\Pi_{c}^{d}\right)$. In order to establish the inequality " $\leq$," take $u \in b d\left(Z^{-}\right)$such that $d\left(0_{n}, b d\left(Z^{-}\right)\right)=\|u\|$ and define $\pi_{1}:=\left(c+u, \sigma_{-u}\right)$, where $\sigma_{-u}:=\left\{\left(a_{t}-u\right)^{\prime} x \geq b_{t}, t \in T\right\}$. In such a way $Z_{1}^{-}=Z^{-}-u$, and then $0_{n} \in b d\left(Z_{1}^{-}\right)$, which entails $\pi_{1} \in b d\left(\Pi_{c}^{d}\right)$. Thus,

$$
\delta\left(\pi, b d\left(\Pi_{c}^{d}\right)\right) \leq \delta\left(\pi, \pi_{1}\right)=\|u\|=d\left(0_{n}, b d\left(Z^{-}\right)\right) .
$$

In the case $\pi \in \operatorname{ext}\left(\Pi_{c}^{d}\right)$ one obtains the desired equality just by replacing int by ext in the previous argument.

The following corollary comes directly from Theorem 1, Proposition 2, and statement (iv) of Theorem 6.

Corollary 1. Let $\pi=(c, \sigma) \in \Pi \backslash \Pi_{\infty}$. Suppose that at least one of the following conditions holds:

(i) $\pi \in \operatorname{cl}\left(\Pi_{s}\right)$;

(ii) $\pi \in \operatorname{ext}\left(\Pi_{s}\right)$ and $d\left(0_{n+1}, b d(H)\right) \neq d\left(0_{n}, b d\left(Z^{-}\right)\right)$;

(iii) $d\left(0_{n+1}, b d(H)\right)=d\left(0_{n}, b d\left(Z^{-}\right)\right) \geq\|c\|$.

Then one has

$$
\delta\left(\pi, b d\left(\Pi_{s}\right)\right)=\min \left\{\delta\left(\pi, b d\left(\Pi_{c}\right)\right), \delta\left(\pi, b d\left(\Pi_{c}^{d}\right)\right)\right\} .
$$

Bounds on $\delta\left(\pi, b d\left(\Pi_{s}\right)\right)$ in terms of $\delta\left(\pi, b d\left(\Pi_{c}\right)\right)$ and $\delta\left(\pi, b d\left(\Pi_{c}^{d}\right)\right)$ may be obtained by reformulating Theorems 3 and 4 . The results in these theorems also provide the inequalities $\delta\left(\pi, b d\left(\Pi_{c}^{d}\right)\right) \leq \delta\left(\pi, b d\left(\Pi_{c}\right)\right)$, for $\pi \in \operatorname{ext}\left(\Pi_{s}\right) \cap \operatorname{int}\left(\Pi_{c}\right)$, and $\delta\left(\pi, b d\left(\Pi_{c}\right)\right) \leq \delta\left(\pi, b d\left(\Pi_{c}^{d}\right)\right)$, for $\pi \in \Pi_{i} \backslash \Pi_{\infty}$. See [5, section 2.4] for a counterpart in the finite case.

The following proposition clarifies the relationship between $\Pi_{s}$ and $\Pi_{c} \cap \Pi_{c}^{d}$ in our context. It shows that although both sets do not coincide, the ill-posedness with respect to the solvability may be identified to the ill-posedness with respect to the primal-dual consistency. We make use of the inclusion

$$
\Pi_{c} \cap \Pi_{c}^{d} \subset \Pi_{b},
$$

which comes from the following standard argument on duality. 
If $\pi \in \Pi_{c} \cap \Pi_{c}^{d}$ and we take any feasible point $\lambda \in \mathbb{R}_{+}^{(T)}$ of the dual problem, i.e., $\sum_{t \in T} \lambda_{t} a_{t}=c$, we have

$$
c^{\prime} x=\sum_{t \in T} \lambda_{t} a_{t}^{\prime} x \geq \sum_{t \in T} \lambda_{t} b_{t} \text { for all } x \in F .
$$

Proposition 11. The following statements hold:

(i) $\operatorname{int}\left(\Pi_{s}\right)=\operatorname{int}\left(\Pi_{c} \cap \Pi_{c}^{d}\right)$;

(ii) $b d\left(\Pi_{s}\right)=b d\left(\Pi_{c} \cap \Pi_{c}^{d}\right)$;

(iii) $\operatorname{ext}\left(\Pi_{s}\right)=\operatorname{ext}\left(\Pi_{c} \cap \Pi_{c}^{d}\right)$.

Proof. We shall prove conditions (i) and (ii), and then (iii) follows. Condition (i) is a consequence of Proposition 3 and Theorem 6, taking into account that int $\left(\Pi_{c}\right) \cap$ $\operatorname{int}\left(\Pi_{c}^{d}\right)=\operatorname{int}\left(\Pi_{c} \cap \Pi_{c}^{d}\right)$.

(ii) If $\pi \in b d\left(\Pi_{c} \cap \Pi_{c}^{d}\right) \subset \operatorname{cl}\left(\Pi_{b}\right)=\operatorname{cl}\left(\Pi_{s}\right)$, where the last equality comes from [4, Thm. 1], then it must be $\pi \in b d\left(\Pi_{s}\right)$, taking into account the previous condition. Assume now that $\pi \in b d\left(\Pi_{s}\right)$ and take $\pi_{r}=\left(c^{r}, \sigma_{r}\right) \in \Pi_{s}$ with $\left\{\pi_{r}\right\}$ converging to $\pi$. By Proposition 1(ii), $c^{r} \in \operatorname{cl}\left(M_{r}\right)$ for each $r$. Then, for each $r$, there exists $\widetilde{c}^{r} \in M_{r}$ with $\left\|\widetilde{c}^{r}-c^{r}\right\| \leq \frac{1}{r}$. Therefore the problem $\widetilde{\pi}_{r}=\left(\widetilde{c}^{r}, \sigma_{r}\right) \in \Pi_{c} \cap \Pi_{c}^{d}$ for all $r$. Since $\left\{\widetilde{\pi}_{r}\right\}$ converges to $\pi$, we have $\pi \in \operatorname{cl}\left(\Pi_{c} \cap \Pi_{c}^{d}\right)$ and then, under the current hypothesis, $\pi \in b d\left(\Pi_{c} \cap \Pi_{c}^{d}\right)$.

Remark 4. In general $\operatorname{cl}\left(\Pi_{c} \cap \Pi_{c}^{d}\right) \subset \operatorname{cl}\left(\Pi_{c}\right) \cap \operatorname{cl}\left(\Pi_{c}^{d}\right)$, but the opposite inclusion does not hold, even in the finite case. Just consider the problem in $\mathbb{R}$ given by $\pi:=\operatorname{Inf}\{-x \mid 0 x \geq 1, x \geq 1\}$. Since $d\left(0_{2}, b d(H)\right)=0=d\left(0, b d\left(Z^{-}\right)\right), \pi \in \Pi_{i} \cap$ $c l\left(\Pi_{c}\right) \cap c l\left(\Pi_{c}^{d}\right)$. However, $\pi \notin b d\left(\Pi_{s}\right)$ (see Proposition 6), and then $\pi \notin c l\left(\Pi_{s}\right)$.

7. The finite case. In the finite case ( $T$ finite), the distances to inconsistency (primal and/or dual) are studied in [5], [10], [16], [18], [19], [20], etc. In particular [10], following the steps of [20], deals with a conic linear system $\sigma=(A, b)$,

$$
\begin{aligned}
\sigma: & b-A x \in C_{Y}, \\
& x \in C_{X},
\end{aligned}
$$

where $C_{X} \subset X$ and $C_{Y} \subset Y$ are closed convex cones in $X$ and $Y$, respectively. $X$ and $Y$ are an $n$-dimensional and an $m$-dimensional normed space, respectively, and the norms in both spaces are represented by $\|\cdot\|$. Here $b \in Y$ and $A: X \longrightarrow Y$ is a linear operator, with norm $\|A\|:=\sup \{\|A x\| \mid\|x\| \leq 1\}$. The parameter space of all systems (14) is endowed with the product norm

$$
\|\sigma\|=\|(A, b)\|:=\max \{\|A\|,\|b\|\} .
$$

This model includes our primal constraint system if $T$ is finite (particularly if $|T|=m$ ) just by taking $C_{X}:=\mathbb{R}^{n}$ and $C_{Y}:=-\mathbb{R}_{+}^{m}$. As a consequence of this fact, finite-dimensional versions of our distances to inconsistency are obtained by applying Theorems 1 and 2 in [10]. Unfortunately the tools developed in [10] and [20] do not apply in our context, in which $Y=\mathbb{R}^{T}$ is not a normed space when $T$ is infinite and arbitrary.

With respect to the aim of relating our results to [10, Thms. 1 and 2] and [20, Thm. 3.5], and if we identify $a \in \mathbb{R}^{n}$ with the linear operator $x \mapsto a^{\prime} x$, a suitable norm to be used in $\mathbb{R}^{n+1}$ would be

$$
\left\|\left(\begin{array}{l}
a \\
b
\end{array}\right)\right\|=\max \left\{\|a\|_{*},|b|\right\} .
$$


Observe that this norm satisfies conditions (3) and (4). Moreover, the norm $\|\cdot\|$ in $X=\mathbb{R}^{n}$ will be arbitrary, whereas in $Y=\mathbb{R}^{m}$ we shall use the norm $\|\cdot\|_{\infty}$.

Specifically, when $T$ is finite, our system $\left\{a_{t}^{\prime} x \geq b_{t}, t \in T\right\}$ can be rewritten in the matrix form $A x \geq b$ (where the $t$ th row of the matrix $A$ is $a_{t}^{\prime}$, and the $t$ th component of the vector $b$ is $b_{t}$ ). Then, and thanks to the fact that in $Y$ we chose the infinite-norm $\|\cdot\|_{\infty}$

$$
\|\sigma\|=\max \left\{\max _{\|x\| \leq 1}\|A x\|,\|b\|\right\}=\max _{t \in T} \max \left\{\left\|a_{t}\right\|_{*},\left|b_{t}\right|\right\}=\max _{t \in T}\left\|\left(\begin{array}{c}
a_{t} \\
b_{t}
\end{array}\right)\right\| .
$$

For system $\sigma$ in (14), [10] presents different mathematical programs, each of whose optimal values provides either the exact distance to inconsistency, denoted by $\rho(\sigma)$, or an approximation of $\rho(\sigma)$ to within certain constants. In particular, if $X=\mathbb{R}^{n}$ and $Y=\mathbb{R}^{m}$, Theorem 2 in [10] establishes, when $\sigma$ is consistent, that $\rho(\sigma)$ coincides with the optimal value of the program,

$$
\begin{array}{ll}
\operatorname{Inf}_{y, q, g} & \max \left\{\left\|A^{\prime} y-q\right\|_{*},\left|b^{\prime} y+g\right|\right\} \\
\text { s.t. } & y \in C_{Y}^{*},\|y\|_{*}=1, q \in C_{X}^{*}, g \geq 0,
\end{array}
$$

where $A^{\prime}$ and $b^{\prime}$ are the transposes of $A$ and $b$, respectively.

In our framework $C_{X}:=\mathbb{R}^{n}, C_{Y}:=-\mathbb{R}_{+}^{m}$ and, writing $\lambda:=-y$, program (17) is equivalent to

$$
\begin{array}{ll}
\operatorname{Inf}_{\lambda, g} & \max \left\{\left\|\sum_{t \in T} \lambda_{t} a_{t}\right\|_{*},\left|\sum_{t \in T} \lambda_{t} b_{t}-g\right|\right\} \\
\text { s.t. } & \lambda \geq 0_{m},\|\lambda\|_{1}=1, g \geq 0 .
\end{array}
$$

By defining $w_{n+1}:=\sum_{t \in T} \lambda_{t} b_{t}-g$, and according to (16), we get another equivalent program:

$$
\begin{array}{ll}
\operatorname{Inf}_{\lambda} & \left\|\left(\begin{array}{c}
w \\
w_{n+1}
\end{array}\right)\right\| \\
\text { s.t. } & \lambda \geq 0_{m},\|\lambda\|_{1}=1, \\
& w=\sum_{t \in T} \lambda_{t} a_{t}, w_{n+1} \leq \sum_{t \in T} \lambda_{t} b_{t} .
\end{array}
$$

In this way we conclude that, for $\sigma$ consistent, the distance to the primal inconsistency $\rho(\sigma)$ coincides with $d\left(0_{n+1}, H\right)$, where $H$ is the hypographical set introduced in section 2. Hence, we recover a partial result in Proposition 2(iv), limited to (primal) consistent problems in the finite case.

On the other hand, the dual problem (10) may be rewritten in the finite case as

$$
\begin{aligned}
\pi^{d}: & \text { Sup } b^{\prime} \lambda \\
\text { s.t. } & A^{\prime} \lambda=c, \\
& \lambda \in \mathbb{R}_{+}^{m} .
\end{aligned}
$$

Assuming that this dual problem is consistent (i.e., $\pi \in \Pi_{c}^{d}$ ), the distance to dual inconsistency $\delta\left(\pi, b d\left(\Pi_{c}^{d}\right)\right)$ is, according to Theorem 3.5 in [20],

$$
\begin{aligned}
& \inf \left\{\|q\| \mid\left\{q=A^{\prime} \lambda-s c, \lambda \geq 0_{m}, s \geq 0,\|\lambda\|_{1}+|s| \leq 1\right\} \text { is inconsistent }\right\} \\
& =\inf \left\{\|q\| \mid q \notin \operatorname{conv}\left(\left\{a_{t}, t \in T ;-c, 0_{n}\right\}\right)\right\}=d\left(0_{n}, b d\left(Z^{-}\right)\right),
\end{aligned}
$$


where we have taken into account the fact that $0_{n} \in Z^{-}$(due to the consistency of $\pi^{d}$ ). Hence, we also recover a partial result in Theorem 6(iv), limited to dual-consistent problems in the finite case.

Finally, let us recall that the specification of inequality (i) in Theorems 3 and 4 for the finite case can be traced from section 2.4 in [5]. Concerning the distance from unsolvable instances to solvability, statements (ii) and (iii) in Theorem 1 are new even in the finite case.

\section{REFERENCES}

[1] M. J. Cánovas, A. L. Dontchev, M. A. López, and J. Parra, Metric regularity of semiinfinite constraint systems, Math. Program., Ser. B, to appear.

[2] M. J. Cánovas, M. A. López, J. Parra, And M. I. Todorov, Stability and well-posedness in linear semi-infinite programming, SIAM J. Optim., 10 (1999), pp. 82-98.

[3] M. J. Cánovas, M. A. López, J. Parra, And F. J. Toledo, Distance to ill-posedness and the consistency value of linear semi-infinite inequality systems, Math. Program., 103A (2005), pp. 95-126.

[4] M. J. Cánovas, M. A. López, J. Parra, and F. J. Toledo, Ill-Posedness with Respect to the Solvability in Linear Optimization, preprint, 2004.

[5] D. Cheung, F. Cucker, And J. PeÑA, Unifying condition numbers for linear programming, Math. Oper. Res., 28 (2003), pp. 609-624.

[6] A. L. Dontchev, A. S. Lewis, And R. T. Rockafellar, The radius of metric regularity, Trans. Amer. Math. Soc., 355 (2002), pp. 493-517.

[7] A. L. Dontchev and R. T. Rockafellar, Regularity and conditioning of solution mappings in variational analysis, Set-Valued Anal., 12 (2004), pp. 79-109.

[8] M. Epelman And R. M. Freund, Condition number complexity of an elementary algorithm for computing a reliable solution of a conic linear system, Math. Program., 88A (2000), pp. 451-485.

[9] M. Epelman And R. M. Freund, A new condition measure, preconditioners, and relations between different measures of conditioning for conic linear systems, SIAM J. Optim., 12 (2002), pp. 627-655.

[10] R. M. Freund And J. R. Vera, Some characterizations and properties of the "distance to ill-posedness" and the condition measure of a conic linear system, Math. Program., 86A (1999), pp. 225-260.

[11] R. M. Freund AND J. R. Vera, Condition-based complexity of convex optimization in conic linear form via the ellipsoid algorithm, SIAM J. Optim., 10 (1999), pp. 155-176.

[12] M. A. Goberna And M. A. LóPez, Linear Semi-infinite Optimization, John Wiley, London, 1998.

[13] M. A. Goberna, M. A. Lopez, And M. I. Todorov, Stability theory for linear inequality systems, SIAM J. Matrix Anal. Appl., 17 (1996), pp. 730-743.

[14] M. A. Goberna, M. A. López, And M. I. Todorov, On the stability of the feasible set in linear optimization, Set-Valued Anal., 9 (2001), pp. 75-99.

[15] D. Klatte and B. Kummer, Nonsmooth Equations in Optimization: Regularity, Calculus, Methods and Applications, Kluwer Academic Publishers, Dordrecht, NL, 2002.

[16] M. A. Nunez, A characterization of ill-posed data instances for convex programming, Math. Program., 91 (2002), pp. 375-390.

[17] M. A. Nunez And R. M. Freund, Condition measures and properties of the central trajectory of a linear program, Math. Program., 83 (1998), pp. 1-28.

[18] J. PEÑA, Understanding the geometry of infeasible perturbations of a conic linear system, SIAM J. Optim., 10 (2000), pp. 534-550.

[19] J. Renegar, Some perturbation theory for linear programming, Math. Program., 65A (1994), pp. $73-91$.

[20] J. RENEGAR, Linear programming, complexity theory and elementary functional analysis, Math. Program., 70 (1995), pp. 279-351.

[21] R. T. Rockafellar, Convex Analysis, Princeton University Press, Princeton, NJ, 1970. 